\title{
A holistic view of mouse enhancer architectures reveals analogous pleiotropic effects and correlation with human disease
}

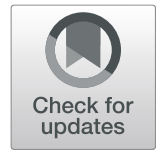

\author{
Siddharth Sethi', Ilya E. Vorontsov ${ }^{2,3}$, Ivan V. Kulakovskiy ${ }^{2,3,4}$, Simon Greenaway' ${ }^{1}$, John Williams ${ }^{1,5,6}$, \\ Vsevolod J. Makeev ${ }^{2,3,7}$, Steve D. M. Brown ${ }^{1}$, Michelle M. Simon ${ }^{1 *}$ and Ann-Marie Mallon ${ }^{1 *}$
}

\begin{abstract}
Background: Efforts to elucidate the function of enhancers in vivo are underway but their vast numbers alongside differing enhancer architectures make it difficult to determine their impact on gene activity. By systematically annotating multiple mouse tissues with super- and typical-enhancers, we have explored their relationship with gene function and phenotype.

Results: Though super-enhancers drive high total- and tissue-specific expression of their associated genes, we find that typical-enhancers also contribute heavily to the tissue-specific expression landscape on account of their large numbers in the genome. Unexpectedly, we demonstrate that both enhancer types are preferentially associated with relevant 'tissue-type' phenotypes and exhibit no difference in phenotype effect size or pleiotropy. Modelling regulatory data alongside molecular data, we built a predictive model to infer gene-phenotype associations and use this model to predict potentially novel disease-associated genes.

Conclusion: Overall our findings reveal that differing enhancer architectures have a similar impact on mammalian phenotypes whilst harbouring differing cellular and expression effects. Together, our results systematically characterise enhancers with predicted phenotypic traits endorsing the role for both types of enhancers in human disease and disorders.
\end{abstract}

Keywords: Super-enhancers, Typical-enhancers, Tissue-specificity, Expression, Phenotypes, Protein-protein interactions, Transcription factors, Gene-phenotype prediction

\section{Background}

Mammalian gene expression and their parallel gene networks are tightly controlled by non-coding regulatory regions such as enhancers, their accompanying transcription factors (TFs), chromatin re-modellers and non-coding RNAs [1]. Large scale programs such as ENCODE [2], FANTOM5 [3] and NIH Roadmap Epigenomics project [4] have generated an initial detailed

\footnotetext{
* Correspondence: m.simon@har.mrc.ac.uk; a.mallon@har.mrc.ac.uk ${ }^{1}$ Mammalian Genetics Unit, MRC Harwell Institute, Oxfordshire OX11 ORD, UK Full list of author information is available at the end of the article
}

exploration of active enhancer and promoter regions in a plethora of tissues and cell types forming a crucial data source for study of regulatory regions. Putative enhancers have been predicted in multiple organisms with $>1$ million estimated in the mouse and human genomes [2, 5-8]. ChIP-Seq analysis of chromatin modification has been widely used to catalogue these potential enhancer and promoter regions, with enhancer loci being enriched in histone $\mathrm{H} 3$ lysine4 monomethylation (H3K4me1) and lacking histone H3 lysine4 trimethylation (H3K4me3), while active enhancer sites have the addition of histone H3 lysine27 acetylation (H3K27ac) 
$[5,9]$. Contrastingly, active promoter regions have an enrichment of H3K4me3 and H3K27ac, and a depletion of H3K4me1 [5, 10]. Although these elements have been comprehensively identified, catalogued and archived, numerous questions still remain on the interpretation of their biological relevance, effect on gene expression, and overall impact on disease causation.

Stringent control of transcription is required for the correct functioning of multicellular organisms, with different regulatory regions occupying different roles; promoters initiate transcription while enhancers control the correct spatio-temporal expression of genes [11]. Looping of the chromatin brings the enhancers close to the promoter regions of their target genes [12-14]. As a result, the enhancers increase the rate of transcription by increasing the number of factors involved in the process. Most important factors among these include the Mediator complex, which is a co-activator complex binding to other TFs and RNA polymerase II [15]; cohesin, which stabilises and sometimes even drives cell-type specific enhancer-promoter communication bridges [15]; and factors important for paused RNA polymerase II release and elongation such as $B R D 4$ [16]. How these interactions and chromatin looping are established remains largely unknown. However, regulatory elements; TFs, chromatin modellers, enhancers and promoters must be in close concert to promote transcription, while their disruption may lead to disease in humans and related phenotypes in model organisms such as mouse [11, $17,18]$. Furthermore, over $90 \%$ of GWAS SNPs associated with human disorders occur within the non-coding regions, with $64 \%$ of the non-coding SNPs in enhancer (H3K27ac positive) regions [19-21]. Similarly, 76\% of non-coding SNPs from GWAS are identified either within DNaseI hypersensitive sites (DHS) or in high linkage disequilibrium with a SNP within DHS [20]. Indeed, the number and scale of putative disease variants identified in the non-coding genome has driven the characterisation of enhancers and their association to pathological states. The pathology of disease in humans is commonly studied in the laboratory mouse, typically by analysing the phenotypes arising from targeted mutations. Phenotyping initiatives like the International Mouse Phenotyping Consortium (IMPC) [22, 23] identify phenotype-genotype associations by producing mouse lines with a protein-coding gene knockout and systematically recording the results from a battery of phenotyping tests for each line. These standardised tests cover a multitude of biological processes and provide consistent descriptions of phenotypes for each functional gene, which can be used in the understanding of human traits and diseases. As with the coding regions of the mouse genome, the study of enhancers and other noncoding regions has been greatly facilitated by CRISPR and on a case-by-case basis we are beginning to understand the roles of enhancers in the susceptibility and pathogenesis of disease [24-30]. However, despite recent progress in the study of the non-coding genome, systematic genotype-phenotype analysis of enhancers and other non-coding regions remains a substantial challenge.

Recently, dense clusters of active enhancers have been recognised as a new class of regulatory element termed super-enhancers (SEs) [31]. These elements spanning large genomic regions are enriched with various chromatin regulators and cofactors such as the Mediator complex, p300, Brd4 and RNA polymerase II [21]. Mediator binding and H3K27ac chromatin marks have been most commonly used to segregate SEs from regular enhancers referred to as typical-enhancers (TEs). Systematic mapping of SEs using H3K27ac chromatin mark across diverse human tissues and cell lines show that SEs regulate genes that define cell identity and drive high expression of their target genes compared to TEs [21, 32-34]. While studies in the mouse genome find similar results, they are currently limited to relatively few tissue types [31, 35-39]. Furthermore, SEs in human cell types have been shown to frequently harbour disease-causing variation [21, 40, 41], while TEs have been considered less important. However, to date there has been no systematic study defining genome-wide functional difference between SEs and TEs, and their relationship to phenotypes.

Here, we systematically identified highly tissue-specific enhancers in 22 mouse tissues, and further classified them into SEs and TEs. Moreover, we linked these enhancers with genes associated with phenotypic effects in the mouse. We find that though SEs drive high totalexpression (aggregated expression of all exons) and tissue-specific expression (tendency of gene to be specifically expressed in a tissue or cell line) of their associated genes, large number of TEs in the genome enable them to contribute greatly to the tissue-specific expression landscape. For the first time our results show both SE and TE associated genes are enriched for relevant phenotypes and diseases in the corresponding tissue-types, and we show there is no significant difference in severity and breadth of phenotypes produced from knockouts of $\mathrm{SE}$ and TE associated genes, indicating the importance of both enhancer types in disease causation. We go on to use regulatory data combined with other molecular characteristics to infer mammalian gene-phenotype associations and identify potential novel pathogenic genes which may be used for further characterisation.

\section{Results}

Systematic profiling of tissue-specific regulatory elements (TSREs) in mouse

To systematically identify potential regulatory elements in the mouse genome, we annotated genome-wide 
chromatin states using a multivariate hidden Markov model called ChromHMM [42]. We constructed the model using three primary histone marks (namely H3K4me1, H3K4me3 and H3K27ac) in 22 mouse epigenomes from ENCODE [2]. These chromatin states can be broadly categorised into active promoter, weak promoter, strong enhancer and weak enhancer states (Additional file 1: Figure S1). Overall, we annotated 923, 791 strong enhancer and 309,581 active promoter annotations (each being $200 \mathrm{bp}$ in length) across the 22 epigenomes (posterior probability of states $\geq 0.95$ ). To validate the accuracy of our predicted promoters and strong enhancers, we compared them to known promoter and enhancer elements in the mouse genome (see methods). The predicted regulatory elements achieved a recall sensitivity of $81.7 \%(18,543 / 22,707)$ for the promoters of protein-coding genes, and $91.2 \%$ (331/363) for enhancers. To accurately identify mouse TSREs, we implemented the previously described TAU algorithm [43, 44 ] to calculate the tissue specificity index $\left(\tau_{\text {reg }}\right)$ of every strong enhancer and active promoter (see methods). In total across 22 mouse tissues, 31\% of all strong enhancers were shown to be highly tissue-specific $\left(\tau_{r e g} \geq\right.$ 0.85 ) and $43 \%$ of active promoters. Both, also show a high degree of positive correlation with DNaseI hypersensitive sites (DHS) in the corresponding tissues (Pearson's correlation, $p<2.2 \mathrm{e}-16$ ), confirming these TSREs are highly tissue-specific (Fig. 1a-b, Additional file 1: Figure S2).

To identify mouse SEs, we used the ROSE algorithm [31] to combine tissue-specific enhancer elements within a span of $12.5 \mathrm{~kb}$ into cohesive units and rank them based on H3K27ac signal which distinguishes them from TEs (Fig. 1c). The enhancer elements within the cohesive units (for both categorised as SEs or TEs) are referred to as constituent enhancers (Additional file 1: Figure S2d). Using this approach, 6.6\% (5082) of all cohesive units (or $24 \%$ of all tissue-specific enhancers) are SEs while $93.4 \%(71,824)$ are TEs (or $76 \%$ of all tissuespecific enhancers) (Additional file 1: Figure S2e). As expected, we found SE cohesive units are occupied on average by $2.4 \mathrm{x}$ H3K27ac and span large genomic regions (median size $=12.4 \mathrm{~kb}$ ) compared to TEs (median size $=0.4 \mathrm{~kb})($ Fig. 1d-e, Additional file 1: Figure S3). The number of constituent enhancers are enriched in SEs compared to TEs (Fig. 1f). Enrichment of H3K4me1 and DHS at SEs is observed to be in agreement with H3K27ac levels (Additional file 1: Figure S4). To determine whether the high levels of histone modification activity at SEs are a consequence of the total genomic length of their cohesive units, we compared the enrichment of H3K27ac and H3K4me1 among their constituent enhancers to TEs. We find that constituent enhancers within SEs show a higher density of H3K27ac and H3K4me1 histone marks compared to TEs (Additional file 1: Figure S5a and S5b), suggesting the increased levels of chromatin activity in SEs is not a consequence of the total genomic length of their cohesive units. A similar trend was identified for RNA polymerase II indicating a potential role of enhancer RNAs (eRNAs) in enhancer activity and gene regulation, as reported in recent studies [45, 46] (Additional file 1: Figure S5c).

SEs have been found to frequently overlap the genes they regulate $[21,31]$. A previous study in murine ESCs identified more than $80 \%$ of SEs and TEs to interact with their nearest active gene [47]. To explore the functional role of enhancers we associated each enhancer element to a potential target gene using a community accepted tool, GREAT [48]. We identified 3617 and 14,791 proteincoding genes associated with SEs and TEs in at least one tissue or cell type, respectively (Additional file 2). The resulting enhancer-gene associations were highly consistent with previously identified topological associated domains (TADs) (96\% in cortex TADs and 93\% in $\mathrm{mESC}$ TADs) [49] (Additional file 1: Figure S6a, Additional file 3). Similarly, $87 \%$ of associations overlapped with computationally derived enhancer-promoter units (EPUs) [6]. As expected, the majority (62.53\% of SEs, $57.25 \%$ of TEs) of the tissue-specific enhancers are located within $50 \mathrm{~kb}$ from the transcription start sites (TSSs) of their associated genes (Additional file 1: Figure S6b-S6d). The predicted SEs, TEs and their associated genes were used for all subsequent analysis.

\section{Typical and super-enhancers can boost tissue-specific gene expression}

Previous studies in human and mouse cell types have shown SEs to be related with highly expressed genes [21], however the studies in mouse were less comprehensive and limited to a few tissues [31, 35, 39, 50]. In addition to this total-expression, a few studies have demonstrated SEs to be associated with tissue-specific gene expression in cell lines. For instance, genes associated with SEs in multiple myeloma cell lines were preferentially expressed in myeloma cells [32]. With the aim of exploring whether this association prevails genome-wide, across multiple tissue types and different enhancers, we examined the impact of these newly identified enhancers in 22 tissues. To inspect this, we utilised ENCODE RNA-Seq data. To effectively identify any common expression patterns between genes, tissues and enhancers, we constructed a dataset formed of genes expressed within a particular tissue, termed gene-tissue pairs, followed by categorisation on their type of enhancer association, hence grouping them into three classes: (1) gene-tissue pairs associated with SEs, referred to as super-enhancer class (SEC); (2) gene-tissue pairs 


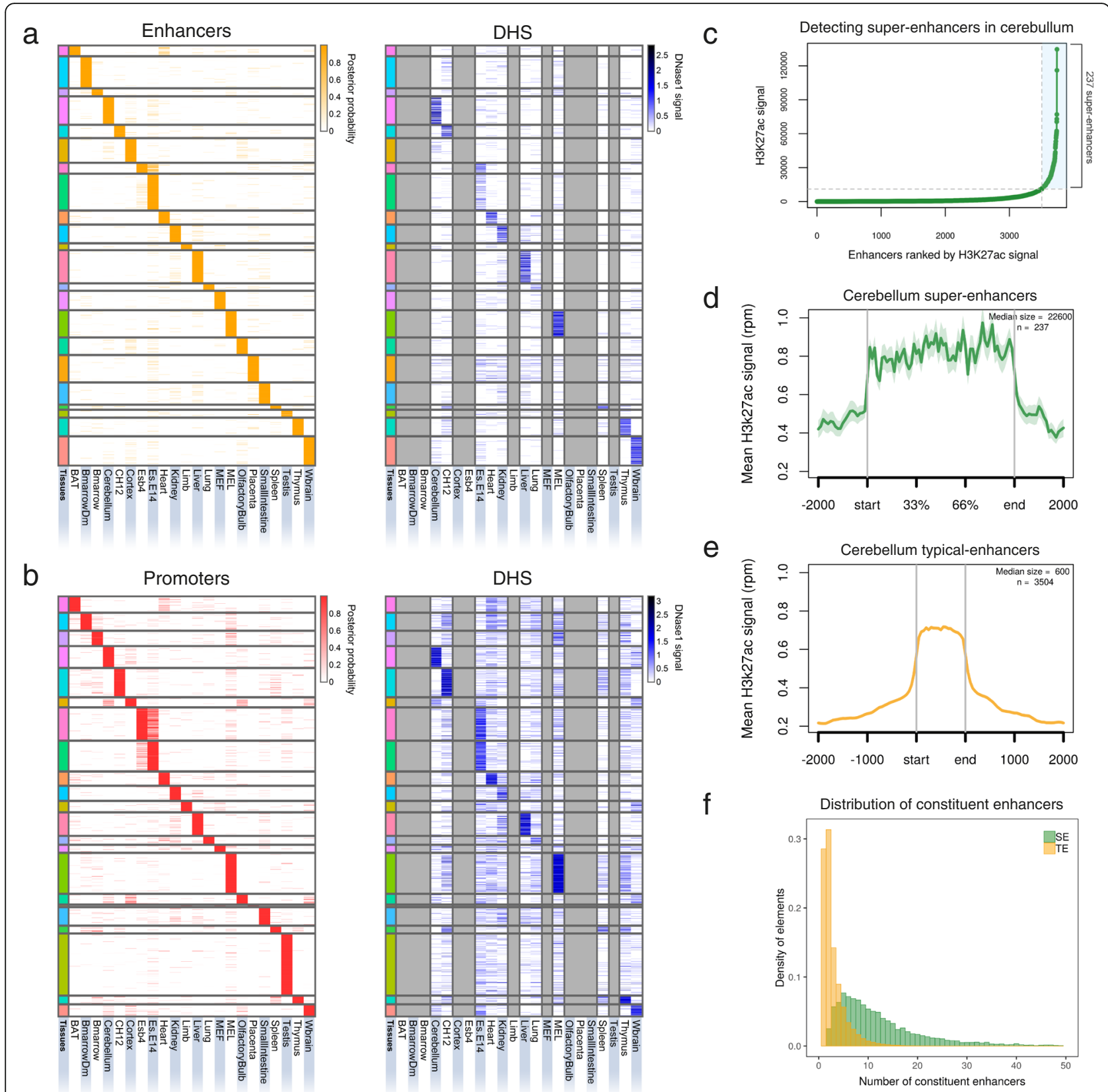

Fig. 1 Overview of TSREs identified in 22 mouse tissues. a Strong enhancers, b Active promoters: Heatmaps showing chromatin state posterior probability of tissue-specific regulatory elements (Taureg $\geq 0.85$ ) (left) and their corresponding DNAse1 signal (right) in every tissue. Each row is a genomic location and columns represent different mouse tissues and cell lines. Grey columns show tissues for which data was not available. The heatmaps have been sorted by the order of the tissues across the columns. (BAT: Brown Adipose Tissue; Bmarrrow: Bone Marrow; BmarrowDm: Bone Marrow derived macrophage; CH12: B-cell lymphoma; Esb4: mouse embryonic stem cells; Es-E14: mouse embryonic stem cell line embryonic day 14.5; MEF: Mouse Embryonic Fibroblast; MEL: Leukaemia; Wbrain: Whole Brain). c Distribution of H3K27ac ChIP-seq signal over cerebellum-specific enhancers stitched together within $12.5 \mathrm{~kb}(n=3741)$. Stitched cohesive units ( $x$-axis) are ranked in an increasing order of their input-normalised H3K27ac signal (reads per million, y-axis). This approach identified 237 SEs (highlighted in blue) and 3504 TEs in cerebellum. d-e Metagene profile of mean H3k27ac ChIP-seq signal across all the SEs and TEs in cerebellum. The profiles are centred on the enhancer regions and the surrounding $2 \mathrm{~kb}$ regions around each enhancer is shown. The length of the enhancer region is scaled to represent the median size of SEs $(22,600 \mathrm{bp})$ and TEs $(600 \mathrm{bp})$ in cerebellum. The shaded area shows the standard error (SEM). $\mathbf{f}$ Distribution of constituent enhancers within SEs and TEs across all 22 tissues. See also Additional file 1: Figure S2-S5 
associated with TEs, referred to as typical-enhancer class (TEC); and (3) gene-tissue pairs associated with weak/ poised enhancers, referred to as weak-enhancer class (WEC).

We found that both SEC and TEC are associated with highly expressed genes in comparison to the WEC (SEC: effect size $(\mathrm{ES})=0.95, p<2.2 \times 10^{-16}$; TEC: $\mathrm{ES}=0.86, p<$ $2.2 \times 10^{-16}$; Wilcoxon Rank Sum Test) but that the SEC appears to have the highest level of total-expression (SEC compared to TEC: ES $=0.56, p<2.2 \times 10^{-16}$ ) (Fig. 2a, Additional file 1: Figure S7a). Likewise, the SEC have higher tissue-specific expression (quantified as $\tau_{\exp \text {-frac }}$, see methods) compared to the TEC ( $\mathrm{ES}=0.62, p<2.2 \times$ $10^{-16}$; Wilcoxon Rank Sum Test) or WEC (ES $=0.96, p<$ $2.2 \times 10^{-16}$ ) (Fig. 2b). To further understand tissuespecific expression of the genes within different enhancer classes, we categorised it into three levels of low, intermediate and high (see methods). We identified, 16.46\% $(690 / 4191)$ of SEC, $4.42 \%(1923 / 43,484)$ of TEC and $3.38 \%(230 / 6795)$ of WEC to have high tissue-specific expression (Fig. 2c, Additional file 1: Figure S7b). Further examination of the high tissue-specific expression category shows the absolute number of genes within the TEC (1923) is notably higher than in the SEC (690) or WEC (230). Overall this data suggests the ratio of genes within the SEC with high tissue-specific expression is at least 4 times larger than the genes within other enhancer classes. However, their absolute number is smaller compared to the TEC which contribute the largest amount (68\%) of enhancer associated tissue-specific expression in the genome (Fig. 2d). This body of work in mouse strengthens the theory that super-enhancers can boost tissue-specific gene expression, while highlighting that high numbers of typical-enhancers, can also boost tissue-specific expression and should not be overlooked.

While identifying SEs we observed they are comprised of a large number of constituent enhancers (Fig. 1f). The average number of constituent enhancers within SEs is 13, compared to 3 in TEs. To this end, we examined whether an increase in the number of constituent enhancers results in an increase in total-expression of their associated genes. To increase the power of this analysis, we combined both the SEC and TEC into a single dataset. We correlated the frequency of the constituent enhancers (total number of constituent enhancers associated with a gene) within the combined dataset with total-expression of their associated gene, which revealed a weak positive correlation (Spearman's correlation $r h o=0.12, p<2.2 \times 10^{-16}$ ) (Additional file 1: Figure S8a). To ensure this observation was not driven predominantly by one class of enhancer, we examined this correlation separately within SEC and TEC, and found no notable difference between the two classes (Additional file 1: Figure S8b and S8c). In contrast, weakenhancer elements show little to no correlation with total- expression (Spearman's correlation rho $=-0.03, p=0.02$ ) of their associated genes (Additional file 1: Figure S8d). Overall this shows that total-expression of a gene modestly increases with an increase in the number of constituent enhancers, indicating a non-additive relationship between them. This suggests that constituent enhancers appear to exert a complex, instead of a simple additive effect on the transcriptional output.

Since a gene could be related to SEs or TEs in multiple tissues, we inspected these multiple gene-enhancer associations for their effect on tissue-specific expression. For this purpose, we assessed the number of distinct tissues, where an enhancer associated with a gene occurs, which we define here as "enhancer tissue-types" (Fig. 2e). A large portion $(\sim 78 \%, 2821$ out of 3617$)$ of the SEC is associated with one enhancer tissue-type, i.e. the genes are associated with SEs from one tissue (Fig. 2f). However, only $27 \%$ (3956 out of 14,791) of the TEC have one enhancer tissue-type, while the remaining $73 \%$ are associated with TEs of two or more tissues (Additional file 4 provides the list of these genes). Furthermore, we see that genes with a higher number of enhancer tissuetypes are associated with low values of $\tau_{\text {exp -frac }}$ (Fig. 2g), hence increasing enhancer tissue-type association increases ubiquitous expression.

We next turned our attention to the genes which are associated with more than one enhancer tissue-type. Since these genes are associated with enhancers in multiple tissues (two or more), we sought to examine what type of enhancer has a higher propensity to adopt an "enhancer usage switch". We define "enhancer usage switch" as the phenomenon where the enhancer usage associated with a gene could differ across multiple tissues. We use the number of constituent enhancers (within SEs or TEs) associated with a gene-tissue pair as a measure of its enhancer usage. The standard deviation of its enhancer usage across the 22 tissues was used to predict the level of "enhancer usage switch". A gene with a large "enhancer usage switch" score refers to an enhancer usage which varies highly across the different tissues. We compared the enhancer usage switch scores between SEC and TEC with multiple enhancer tissue-types, which shows that SEC exhibit significantly higher enhancer usage switch across the tissues (ES $=0.89, p<2.2 \times$ $10^{-16}$; Wilcoxon Rank Sum Test) (Additional file 1: Figure S9). The genes with a high enhancer usage switch score for SEC include: Ntm, Grm4, Foxa2, and Max, whereas the genes with a high enhancer usage switch score for TEC include: Csmd1, Ntrk3, Grin2a and Opcml (Additional file 1: Figure S10; Additional file 5). Overall, this analysis shows that both SEC and TEC display enhancer usage switch, but SE usage of a gene varies significantly more across different cell- and tissue-types compared to TE. 
a

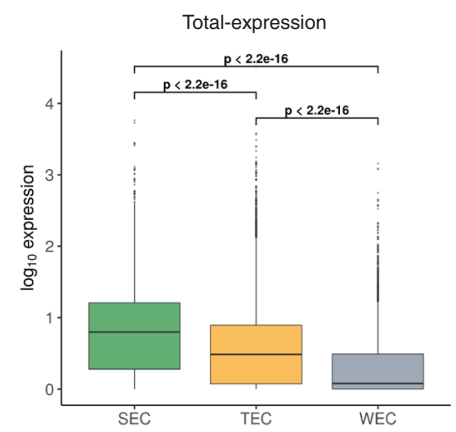

b

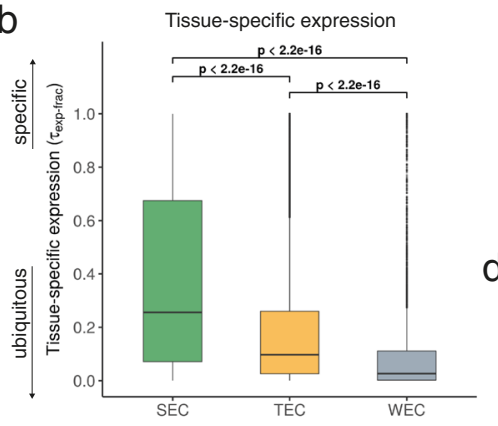

e

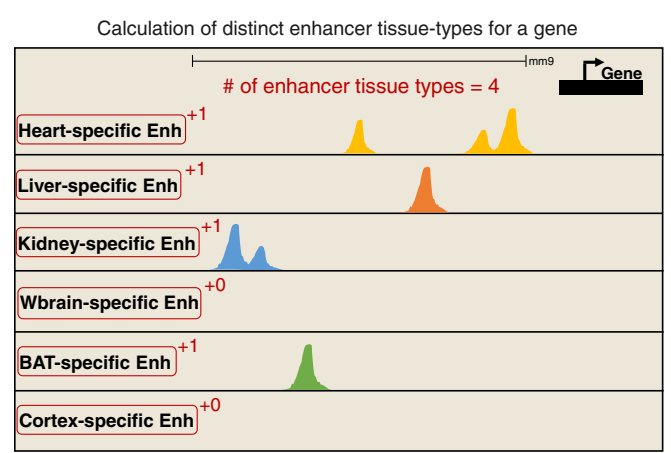

f

Associated with SE Not associated with SE

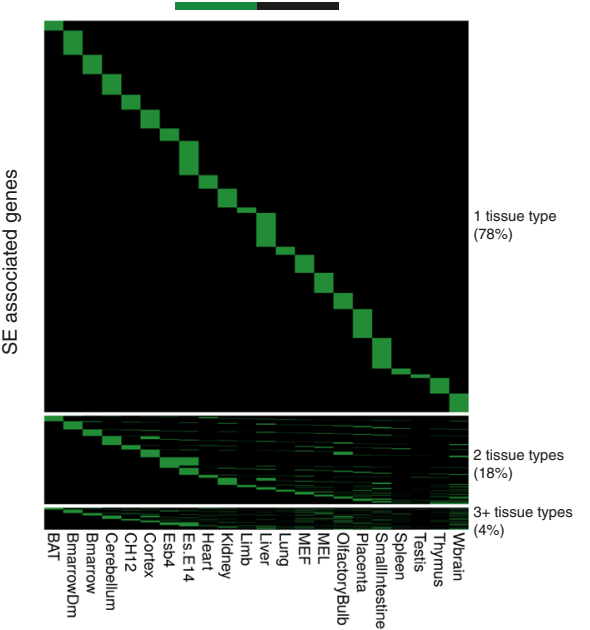

C Genome-wide enhancer activity and tissue-specific expression profile

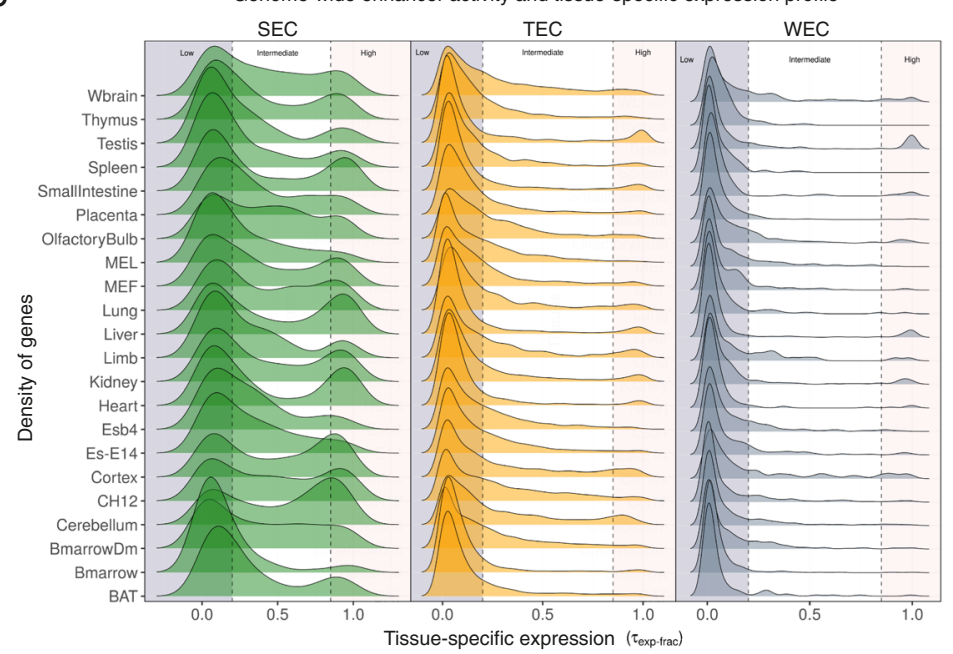

d Contribution of enhancer classes towards tissue-specific expression

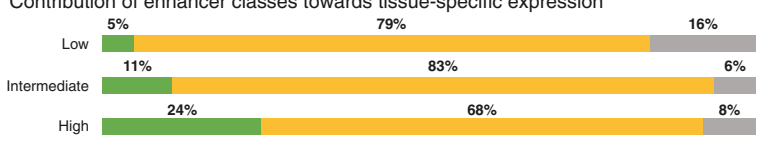

Enhancer associated genes

g

$\rightleftharpoons$ SEC

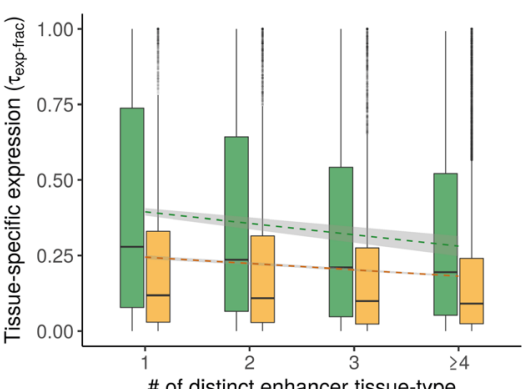

TEC

Associated with TE Not associated with TE

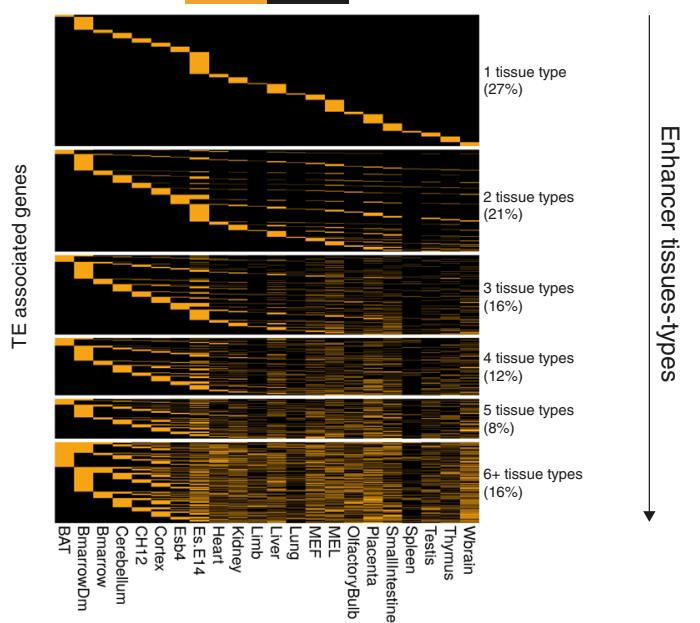

Fig. 2 (See legend on next page.) 
(See figure on previous page.)

Fig. 2 SEs promote high transcriptional activity and drive tissue-specific expression in mouse. a Box plot showing the total-expression (in logtransformed RPKM) of different enhancer classes across 22 tissues. Each box plot shows the median, middle bar; interquartile range, the box; whiskers, 1.5 times the interquartile range. b Box plot showing the tissue-specific expression of different enhancer classes across 22 tissues. The $p$ values were calculated using Wilcoxon Rank Sum Test. c Distribution of genes within tissue-specific expression categories (low, intermediate and high) in different enhancer classes. Y-axis for each tissue displays the density of genes scaled across the tissues, but not across the enhancer classes. d Contribution of each enhancer class (in percentage) towards the total number of enhancer associated genes in the genome, categorised by their tissue-specific expression. e A schematic to illustrate the calculation of distinct enhancer tissue-types for each enhancerassociated gene. The number of distinct tissue types of various enhancers associated with the gene of interest are added to compute the number of enhancer tissue-types for a gene. $\mathbf{f}$ Heatmaps showing the number of enhancer tissue-types in SEC and TEC. Each row is an enhancer associated gene and columns represent its association with enhancers across 22 tissues and cell types. $\mathbf{g}$ Box plot showing the correlation between the number of enhancer tissue-types and tissue-specific expression of SEC and TEC. The trend lines (green: SEs; orange: TEs) were calculated using linear regression. See also Additional file 1: Figure S7 and S8

\section{Enhancers drive phenotype and disease causation}

Previous studies have identified SEs to be associated with genes that regulate cell identity and are therefore unlikely to be involved in a housekeeping role [21, 31]. To increase our understanding of the functional role of $\mathrm{SE}$ and TE associated genes we performed Gene Ontology (GO) enrichment analysis in 22 mouse tissues. Genes associated with SEs belonging to the SEC category are enriched for transcription factor binding activity $\left(p=10^{-10}\right)$, regulation of cell development $\left(p=10^{-16}\right)$ and regulation of cell differentiation $\left(p=10^{-23}\right)$ (Additional file 6). The breadth of this analysis demonstrates novel cell identity associations in unexplored tissues in the mouse. As expected, these are also important in the control and regulation of tissue or cell identity. Some examples of these novel SE associated genes include Ucp1 (responsible for generating body heat in mammals [51]) in brown adipose tissue; Gata4 (critical for heart development and cardiomyocyte regulation [52]) in heart; Cxcr2 (regulates the emigration of neutrophils from bone marrow [53]) in bone marrow; and Rbfox3 (splicing regulator of neuronal transcripts $[54,55]$ ) in cerebellum. On the other hand, TEC appear to have different enrichments in GO analysis and are linked with genes involved in nucleotide and protein containing-complex binding $\left(p=10^{-6}\right)$, cellular protein localisation $\left(p=10^{-7}\right)$ and cell morphogenesis $\left(p=10^{-5}\right)$. Furthermore, TEC is significantly enriched for housekeeping genes $(p=2.7 \times$ $10^{-11}$, Odds Ratio $(\mathrm{OR})=1.49,95 \%$ Confidence Intervals (CI) $[1.32,1.68])$, while SEC is depleted $(p=0.012$, OR $=$ $0.82,95 \%$ CI $[0.69,0.98])$.

To further explore the regulatory function of enhancers, we investigated mouse phenotypes and human diseases associated with genes within SEC and TEC (see methods). Significant enrichment in both phenotypes and disease ontology terms in the corresponding tissue types was identified (Fig. 3, Additional file 7), suggesting a strong relationship between both SEC and TEC and resulting pathological outcomes (disease causation). For instance, genes associated with cerebellum-specific enhancers are enriched for phenotypes such as impaired coordination $\left(\mathrm{q}=4.83 \times 10^{-8}\right)$ and abnormal synaptic transmission $\left(q=2.46 \times 10^{-7}\right)$, and diseases such as bipolar disorder $\left(\mathrm{q}=8.52 \times 10^{-7}\right)$ and unipolar disorder $\left(q=6.26 \times 10^{-5}\right)$. Similarly, genes related to heartspecific enhancers are enriched for phenotypes like abnormal cardiac muscle contractility $\left(q=9.05 \times 10^{-16}\right)$ and diseases like cardiomyopathy $\left(q=5.45 \times 10^{-14}\right)$ (Fig. 3). In addition, enrichment of blood-related cancers (such as Hodgkin Disease, $\mathrm{q}=1.90 \times 10^{-12}$; T-cell Leukemia, $\mathrm{q}=1.41 \times 10^{-5}$ ) in $\mathrm{CH} 12$ enhancer associated genes is consistent with the idea that oncogenes are placed under the effect of strong enhancers during cancer development leading to over-expression of these genes $[32,56]$. On the other hand, the WEC display either an insignificant or a weak association with phenotypes in majority of the tissues (Additional file 1: Table S1).

However, there is a marked difference in the expression patterns of SEC compared to TEC, which is not observed in their relationship with phenotypes. We explored this dichotomy further by comparing the phenotyping data from knockout mouse lines of genes in SEC and TEC across all tissues within the IMPC data. We reasoned that if $\mathrm{SE}$ associated genes are predominantly related to phenotype occurrence, their associated gene knockouts would cause a more severe phenotype condition (a phenotype with an increased effect size) relative to knockouts of other genes (such as those associated with TEs). We compared several standardised phenotyping procedures within the IMPC and observed a significant difference in severity only for acoustic startle and pre-pulse inhibition ( $\mathrm{ES}=-0.63, p=0.001$ ) (Fig. 4). However, for the majority of the procedures, we observed no significant difference in severity of phenotypes between SEC and TEC (Open field test, $\mathrm{ES}=0.19, p=$ 0.13 ; Grip strength, $\mathrm{ES}=0.19, p=0.55$; DEXA, ES $=-$ $0.02, \quad p=0.75$; Heart weight, $\mathrm{ES}=0.16, \quad p=0.63$; Hematology, ES $=0.16, p=0.1$ ). Next, we sought to examine the breadth of the phenotypes associated with SEC and TEC. For this purpose, we computed the number of top-level phenotype ontology terms associated 


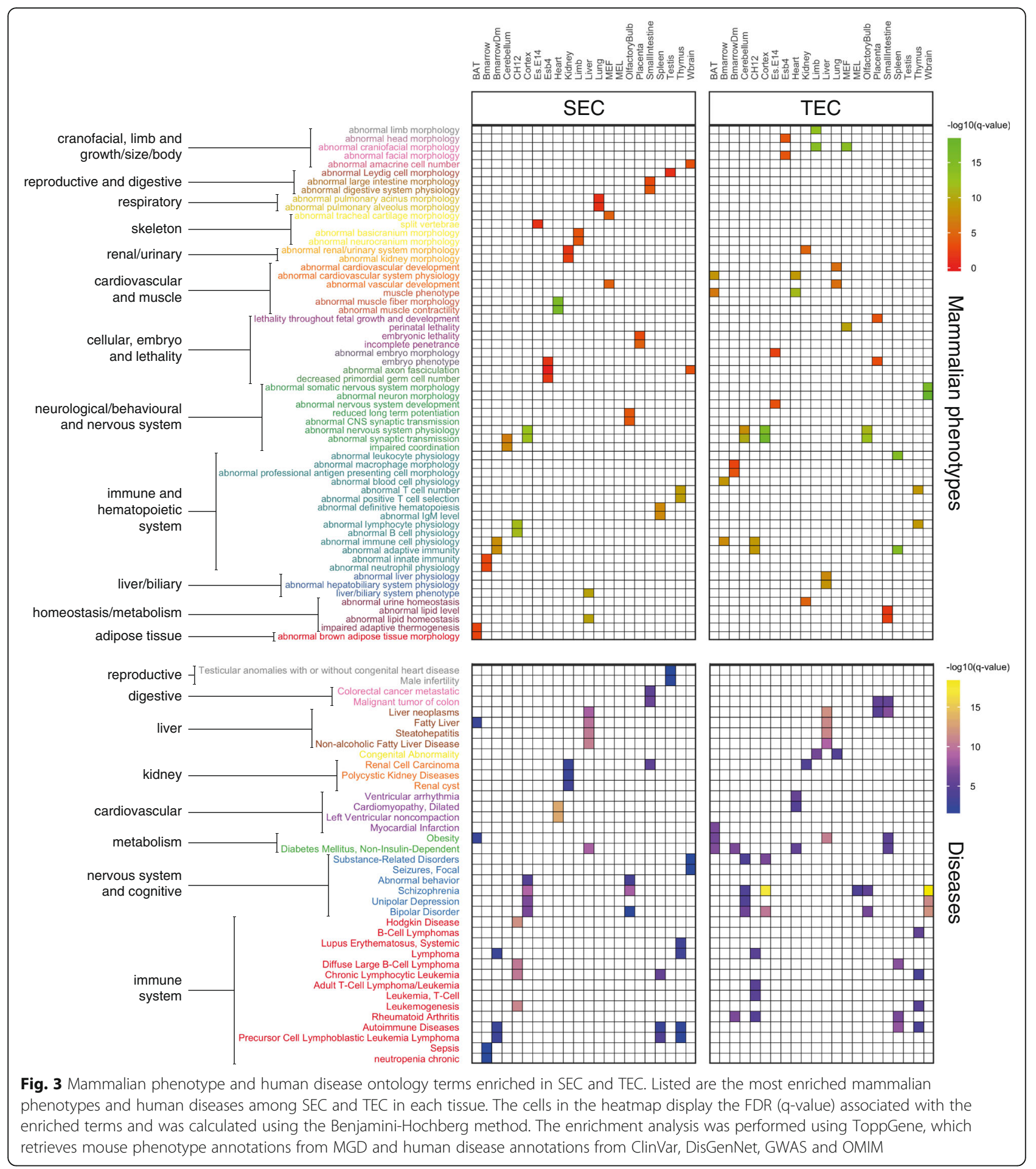

with SE and TE associated gene knockouts from IMPC (Additional file 1: Figure S11). No notable difference is observed in the breadth of phenotypes between SEC and TEC (ES $=0, p=0.42$ ), indicating both SE and TE associated gene knockouts are likely to produce comparable number of phenotypes and therefore, have similar pleiotropic effects. Furthermore, we explored the mouse essential genes by retrieving all the genes from IMPC which generate a lethal knockout [57] to examine if the SEC is enriched with lethality. There is no enrichment of lethal genes among $\operatorname{SEC}(p=0.24, \mathrm{OR}=1.08,95 \% \mathrm{CI}$ $[0.88,1.30])$ and TEC $(p=0.83, \mathrm{OR}=0.93,95 \% \mathrm{CI}[0.79$, 1.09]). Finally, using GTEx data, we compared the number of expression quantitative trait loci (eQTLs) 


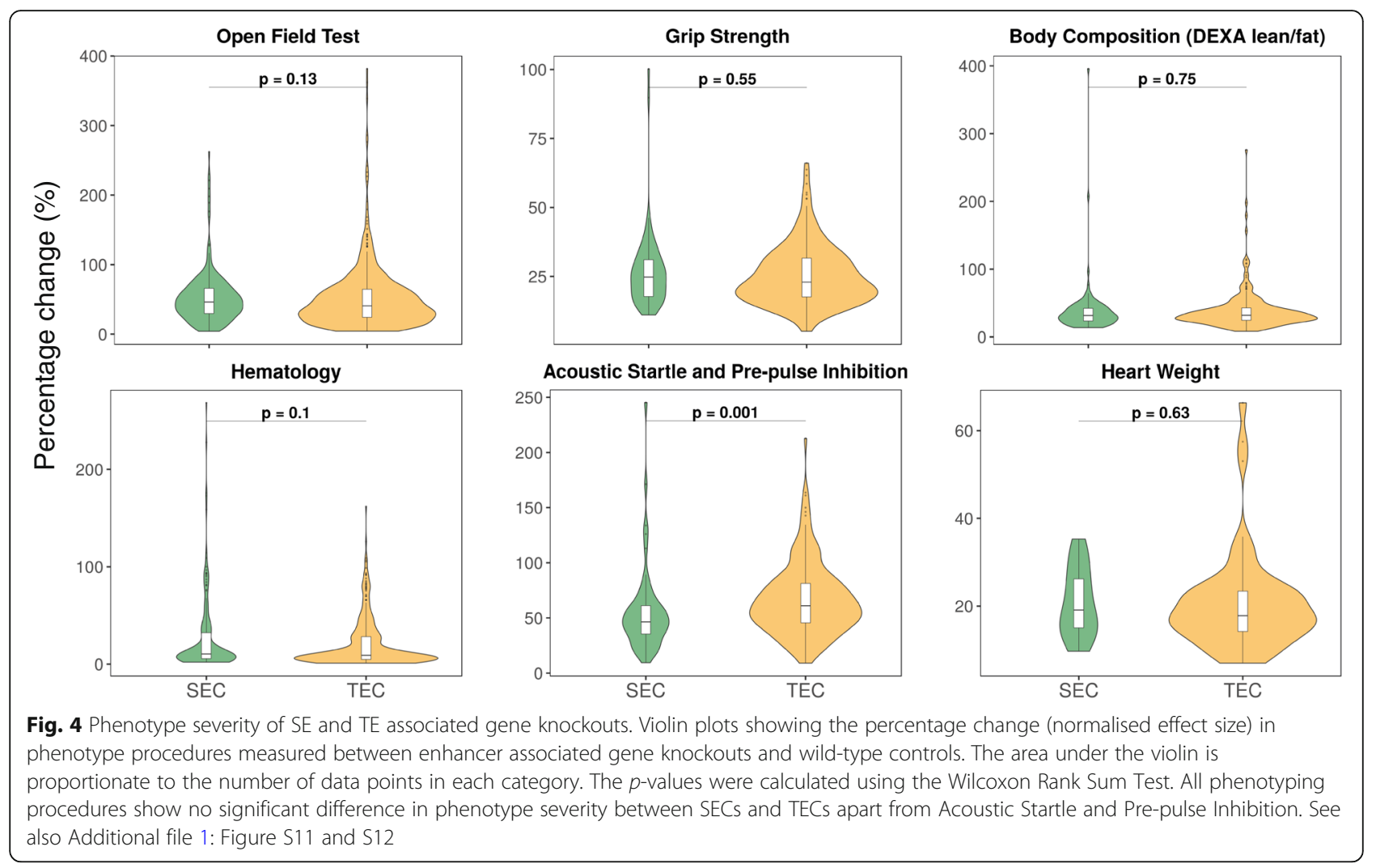

associated with SEC and TEC and observed no significant difference in the number of cis-eQTLs associated with SEC and TEC (ES $=0, p>0.56$; Wilcoxon Rank Sum Test) (Additional file 1: Figure S12). Overall these results highlight that tissue- and cell-specific relevant traits are associated with both SEs and TEs associated genes.

\section{Enhancer associated genes are connected in a dense interactome}

Having shown that enhancer associated genes are enriched for tissue-specific traits, we hypothesised that the proportion of these with no prior phenotypic annotations related to the tissue maybe involved in diseasecausing pathways. To identify novel disease-associated genes, we first analysed the protein-protein interactions (PPI) among enhancer-associated genes in each of the 22 tissues, using the STRING database [58]. Then in each network, we identified the genes currently known to be associated with the corresponding tissue-type phenotypic annotations from MGD [59], while the genes with no-prior phenotypic information were labelled as 'novel'. For each tissue, both the known and unknown disease genes (referred to as known and novel respectively) in the PPI network of enhancer-associated genes are observed to be connected in a remarkably dense interactome (Fig. 5, Additional file 1: Figure S13).
Interestingly, the novel genes (blue nodes) are highly connected with the phenotype-associated genes (pink nodes), suggesting a potential functional relationship between them. Simulating these PPI networks with random protein-coding genes showed that novel genes connect significantly more with known phenotype-associated genes, compared to randomly added genes $(p \leq 0.016$, except thymus $p=0.056$ ) (Additional file 1: Figure S14). This outcome demonstrates enhancer associated genes to be potentially engaged in the same functional pathway as the known phenotype genes and therefore, could also be linked with the corresponding phenotypes and ultimately disease causation.

\section{Preferential transcription factor binding in super- enhancers}

Enhancer regions contain many binding sites for TFs which contribute to important tissue-specific functions by regulating the target genes [60]. To investigate transcription factor binding activity within SEs and TEs, with the aim of identifying potential key regulators in each tissue, we used publicly accessible ChIP-Seq data for mouse TFs. For many TFs, the information available on their specific binding in various cell types is rather sporadic, thus we flattened all available ChIP-Seq peaks for each TF into single binding profiles referred to as "cistrome" (see methods). Next, for each cell type, we systematically 


\section{BAT}

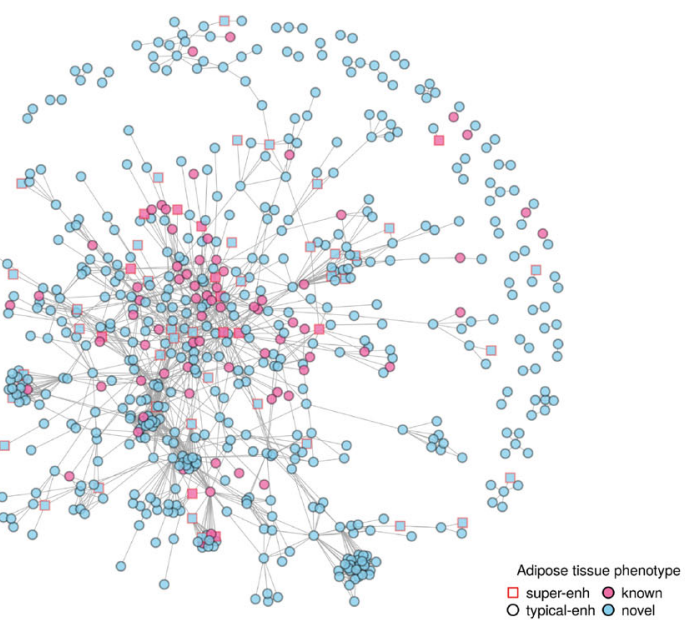

\section{Cerebellum}

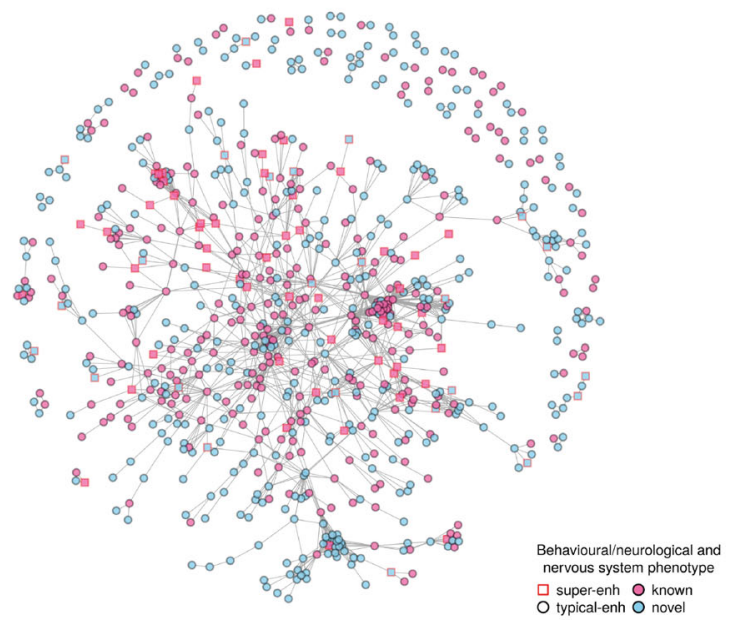

\section{Kidney}

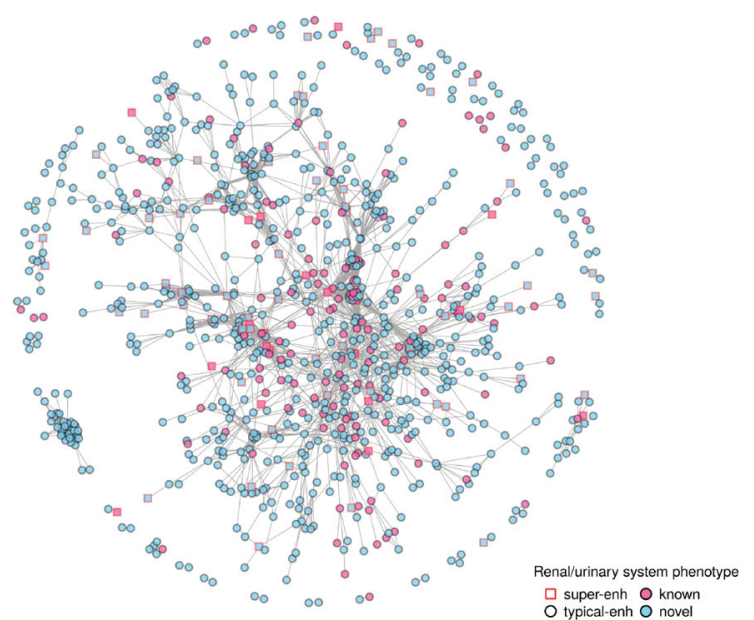

\section{BmarrowDm}

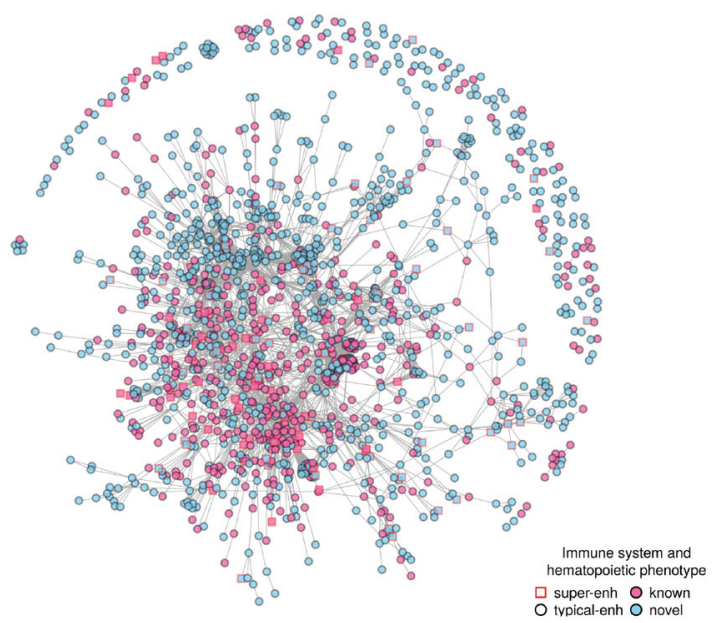

\section{Heart}

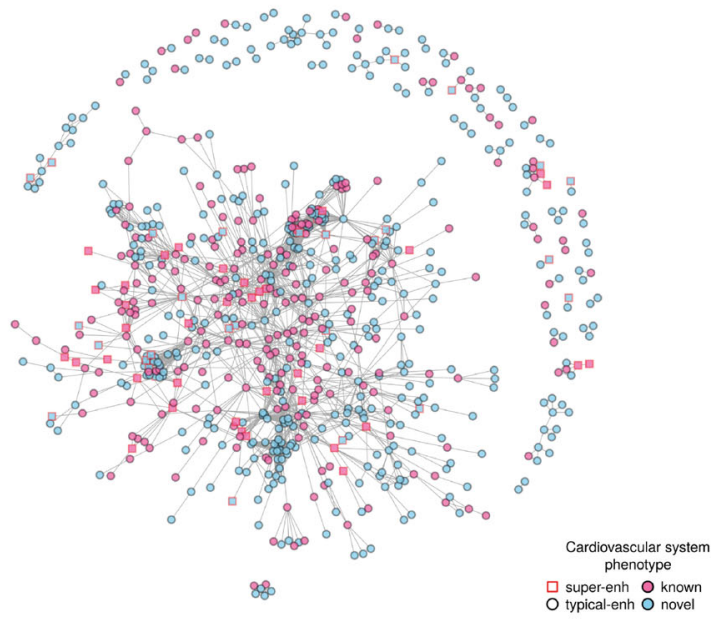

\section{Liver}

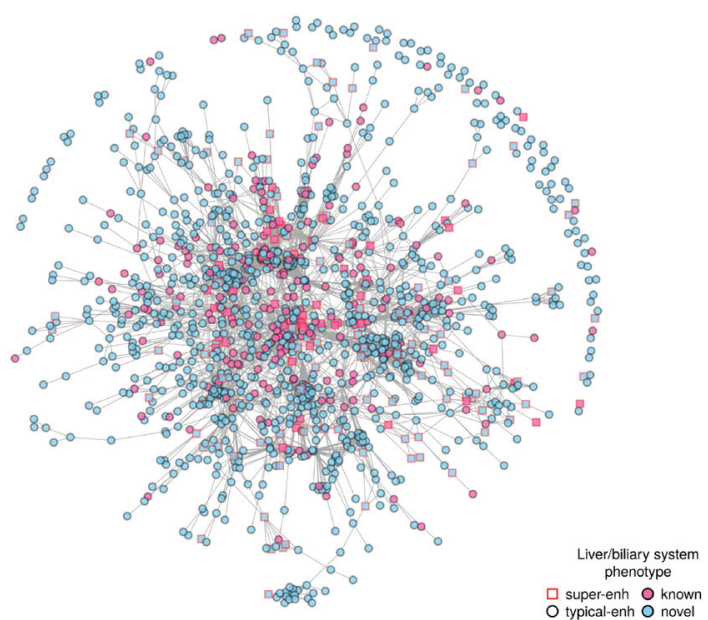

Fig. 5 (See legend on next page.) 
(See figure on previous page.)

Fig. 5 Enhancer associated genes are connected in a dense interactome. The networks display protein-protein interaction maps of enhancer associated genes. Nodes in each network represent enhancer associated genes and edges represent potential protein-protein interactions. Genes associated with tissue-type relevant phenotypes are highlighted in pink and the shape of the node displays SE and TE associated genes (squares: SEC, circles: TEC). See also Additional file 1: Figure S13 and S14

identified TFs, for which cistrome peaks significantly colocalised with their corresponding active enhancers.

First, we found that TFs which have significant colocalisation with enhancer elements include regulators known to be implicated in the corresponding tissuespecific regulation (Fig. 6). For example, Spi1, with cistrome peaks colocalized with bone marrow enhancers, is implicated in myeloid and B-lymphoid cell development [61]; Gata4, with cistrome peaks colocalized with heart enhancers, is involved in myocardial differentiation and function [62]; and Dmrt1, with cistrome peaks colocalized with testis enhancers plays a key role in male sex determination and differentiation [63]. Overall, we observed cistrome peaks of 214 TFs (509 TF-tissue pairs) to significantly colocalise with TEs (with $\mathrm{OR}>1$; corrected $p$-value $<10^{-3}$ ) and 113 TFs (148 TF-tissue pairs) with SEs across all tissues and cell types (Additional file 8). The $214 \mathrm{TFs}$ colocalised with TEs included all the 113 TFs identified for SEs. Second, we observed that some TFs with cistrome peaks significantly colocalised with enhancers are expressed in a tissue-specific manner in the corresponding tissues (Additional file 1: Figure S15a). In total, we identified 56 such TFs with highly tissue-specific expression $\left(\tau_{\text {exp }- \text { frac }}>0.85\right)$ and significant colocalisation with corresponding TEs, and 29 TFs with SEs across all tissues and cell types. Examples of such TFs include Hnf6 in liver $\left(\tau_{\text {exp }- \text { frac }}=1\right)$, Nkx2-5 in heart $\left(\tau_{\exp -\text { frac }}=1\right)$, Gata1 in MEL cells $\left(\tau_{\text {exp }}\right.$ - frac $=0.93)$ and Neurog2 in brain $\left(\tau_{\text {exp }}\right.$ frac $\left.=0.98\right)$.

Overall, TF cistrome peaks were identified to significantly colocalise with both SEs and TEs, but a greater number of TFs were identified to colocalise with TEs compared to SEs. This could be explained by the relatively large number of TEs in the genome. To investigate this further, for each TF with significant enhancer localization, we computed their transcription factor binding site (TFBS) density in SEs and TEs. The TFBS density could be defined as a measure of TFBS clustering in SEs or TEs (see methods). To summarise our analysis, we counted the number of TF-tissue pairs which have significantly greater TFBS density in SEs compared to TEs, and vice-versa for TEs. Overall, we find that SEs have more TF-tissue pairs with higher TFBS density compared to TEs (Additional file 1: Figure S15b). Altogether, this data indicates that although TEs are more often colocalised by TF cistrome peaks, frequency and degree of TFBS clusters is higher in SEs.

\section{Combinatorial learning approach for phenotype prediction}

Our findings show mouse enhancer associated genes are correlated to a great extent with tissue-specific gene expression as well as phenotypes. We explored the utilisation of this dataset to infer mammalian gene-phenotype associations as has previously been done for proteinprotein interaction (PPI) and gene expression data [6466]. We implemented the random forest classifier to predict gene-phenotype associations from 13 different phenotypic domains, where each domain is relevant to at least one tissue type in our dataset. For this learning approach, we extracted gene features from TSRE profiles, expression data, transcription factor binding sites and protein-protein interaction data in 22 mouse tissues (Fig. 7a) (see methods). For the purpose of training this random forest classifier and maximising its learning process, we combined the SE and TE dataset together and used their constituent enhancers (or tissue-specific enhancers) to calculate the enhancer-associated gene feature. We first trained a random forest classifier on a subset of protein-coding genes using a combination of various gene features as predictor variables and the top level mammalian phenotype terms from MGD as the response variable (true positives), while genes not associated with a phenotype in MGD were considered as true negatives. This model was used to predict genephenotype associations in the remaining set of genes not used in the training of the model.

By integrating various features together, 10 combinations were formed, constructing 10 distinct classifiers for each phenotypic domain. The predictive power of each classifier was assessed by generating Receiver Operating Characteristic (ROC) and precision-recall (PR) curves based on 5-fold cross validation, repeated 10 times with different seeds. The classifier trained on all the gene features combined achieved the best performance with a mean AUC-ROC of 0.78 and AUC-PR of 0.27 across all the phenotype domains (Fig. 7b, Additional file 1: Figure S16, Additional file 9). However, high precision recall rates (AUC-PR > 0.35) are observed in phenotypes with a high number of known mammalian phenotype annotation counts in MGD (such as behavioural/neurological, nervous system, cardiovascular, immune and hematopoietic system, see Additional file 1: Figure S17). Focusing on predicting gene-phenotype associations within the nervous system domain, the classifier trained on all the gene features achieved the greatest mean AUC-ROC of 0.80 and 


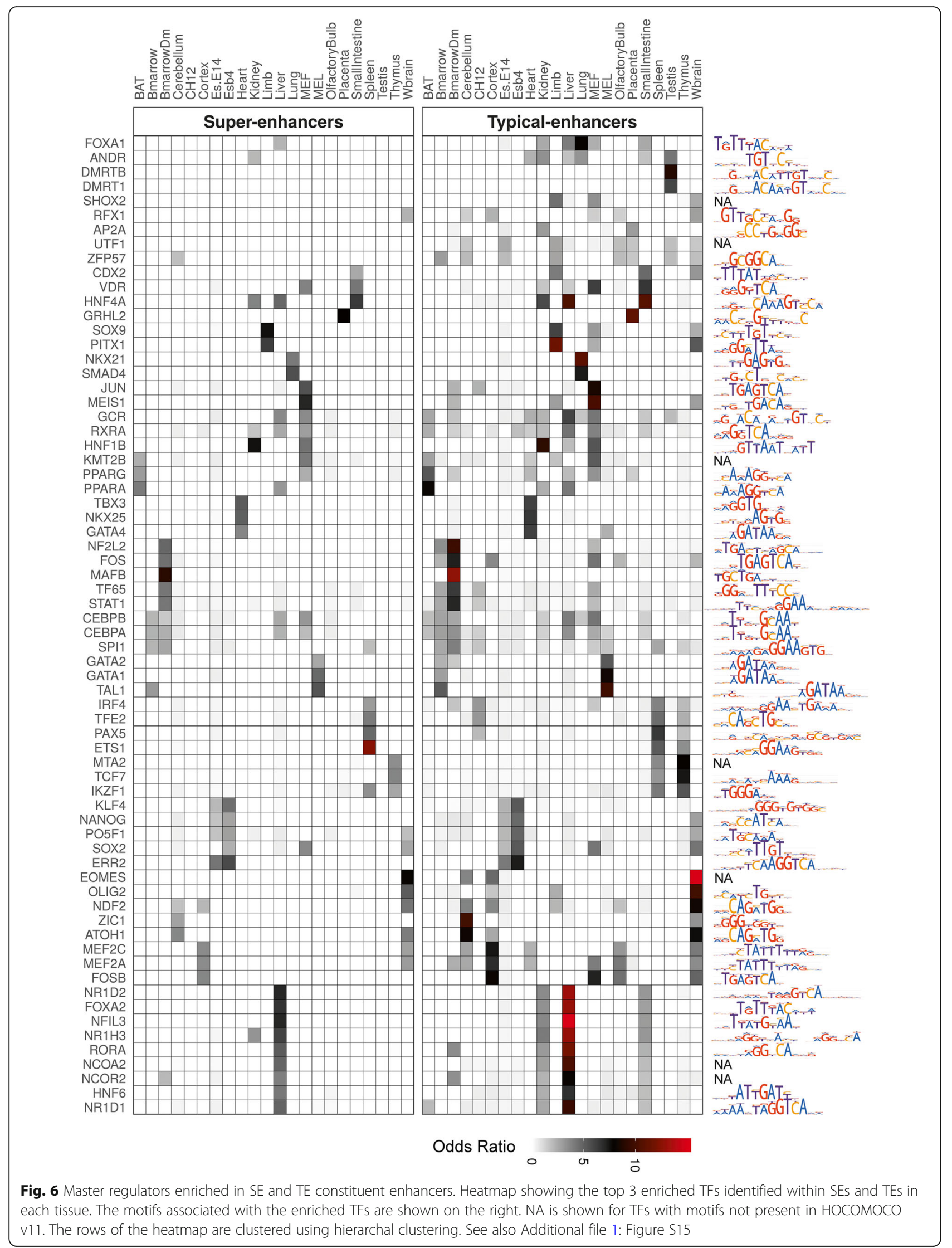


a

\begin{tabular}{|c|c|c|c|c|}
\hline Data & Feature & Description & $\mathbf{n}$ & Symbol \\
\hline \multirow{3}{*}{$\begin{array}{l}\text { Regulatory } \\
\text { elements }\end{array}$} & $\begin{array}{l}\text { Tissue-specific enhancer } \\
\text { profiles }\end{array}$ & $\begin{array}{l}\text { Sum of posterior probabilities for all tissue-specific strong } \\
\text { enhancers associated in each tissue }\end{array}$ & 22 & \multirow{2}{*}{ - TSRE } \\
\hline & $\begin{array}{l}\text { Tissue-specific promoter } \\
\text { profiles }\end{array}$ & $\begin{array}{l}\text { Sum of posterior probabilities for all tissue-specific active } \\
\text { promoters associated in each tissue }\end{array}$ & 22 & \\
\hline & $\begin{array}{l}\text { Transcription factor } \\
\text { binding }\end{array}$ & $\begin{array}{l}\text { Enrichment of motifs within cistrome regions overlapping } \\
500 \text { bp upstream and } 100 \text { bp downstream of TSS }\end{array}$ & 297 & TF \\
\hline \multirow{3}{*}{$\begin{array}{l}\text { Protein-protein } \\
\text { interactions } \\
\text { (PPI) }\end{array}$} & $\begin{array}{l}\text { PPI with genes } \\
\text { associated with } \\
\text { enhancers }\end{array}$ & $\begin{array}{l}\text { PPI score of a gene within tissue-specific enhancer } \\
\text { network }\end{array}$ & 22 & \multirow{2}{*}{ TSRE_PP } \\
\hline & $\begin{array}{l}\text { PPI with genes } \\
\text { associated with } \\
\text { promoters }\end{array}$ & PPI score of a gene within tissue-specific promoter network & 22 & \\
\hline & $\begin{array}{l}\text { PPI with genes } \\
\text { associated with the } \\
\text { phenotype }\end{array}$ & PPI score of a gene within a phenotype associated network & 1 & PPI \\
\hline Expression & Expression profiles & Expression of a gene in each tissue & 22 & Exp \\
\hline
\end{tabular}

b

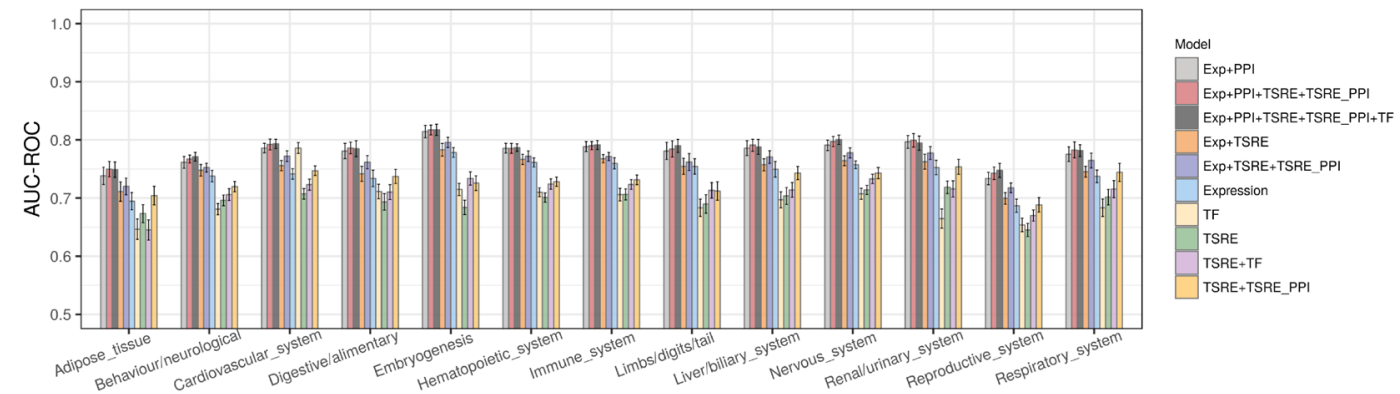

Mammalian phenotypes

C

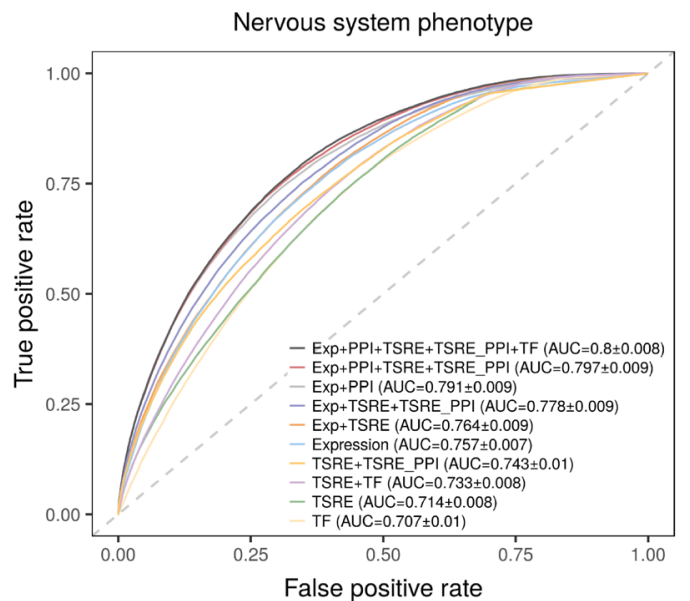

d

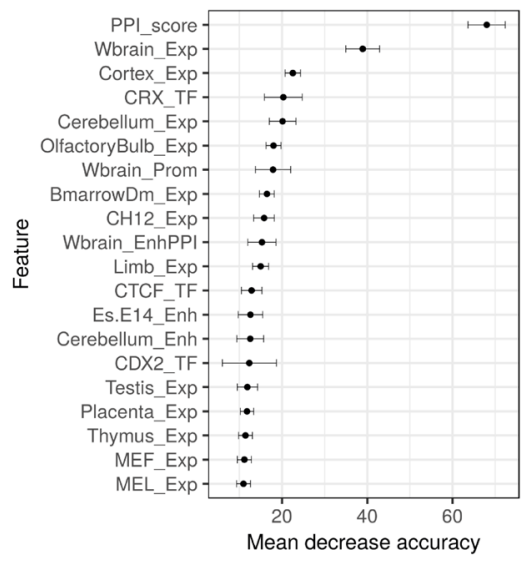

Fig. 7 Predicting gene-phenotype associations in mouse. a Summary of the various gene features (grouped according to their data sources) used to train the random forest classifier to predict gene-phenotype associations. b Bar plot comparing the predictive power of different random forest classifiers across various phenotypes. Error bars denote standard deviation. The classifier trained on all gene features performs the best for majority of the phenotype domains. c Receiver operating characteristic (ROC) curves comparing the performance of 10 random forest classifier models applied to predict genes associated with nervous system phenotype. $\mathbf{d}$ Feature importance chart of the best performing model (Exp + PPI + TSRE+TSRE_PPI + TF) showing the top 20 predictor variables important in nervous system phenotype predictions, as measured by the mean decrease in accuracy ( $x$-axis). The PPI feature was identified to be the most important in predicting genes associated with nervous system phenotype, followed by expression in whole brain and cortex. Exp: expression; Enh: enhancer; Prom: promoter; TF: transcription factor. See also Additional file 1: Figure S16 and S17 
AUC-PR of 0.42 (Fig. 7c). The PPI score with genes known to be associated with nervous system phenotype was identified to contribute the most in predicting nervous system gene-phenotype associations, followed by expression data in whole brain and cortex (Fig. 7d). In fact, PPI data is the most informative and the main contributor to the performance of these classifiers in all the 13 phenotypes. While the models trained solely on regulatory features have limited predictive power, they improved the performance of models when integrated with other features, suggesting regulatory data are a useful addition for modelling mammalian phenotypes.

In order to evaluate the validity of the predictions from the model, we investigated the novel genephenotype predictions made by these classifiers. The predictions classified as incorrect are based on the current knowledge of gene-phenotype associations, but it is possible that there are no, or little, prior knowledge about particular gene function, and thus are novel. This also leads to undermining the true predictive power of a classification model. For such reasons, the top falsepositive predictions are most interesting as they could provide new hypotheses about gene function. To systematically examine the top false-positive predictions (prediction score $\geq 0.90$ ) in each phenotype domain, we used the Open Targets Platform [67] and the DisGeNET discovery platform [68] which links potential novel genes to diseases via evidence based on genetic associations, somatic mutations, animal models, expression, pathways, drugs and text mining from literature. We identified that $\sim 75 \%$ (495/659) of the false-positive predictions examined (see methods) with Open Targets and 63\% (338/ 539) with DisGeNET could be potentially associated with the corresponding disease (Additional file 1: Figure S18) and hence, could serve as potential novel disease targets. For example, out of the 76 top scoring falsepositives (prediction score $\geq 0.90$ ) examined for nervous system phenotype, 72 could be associated with nervous system disease $\left(p=5.00 \times 10^{-9}\right)$ based on evidence integrated from a range of data sources by Open Targets platform. Additional file 10 provides these novel predictions for each phenotype and the evidence supporting their association with the corresponding diseases.

\section{Discussion}

Regulatory elements have been identified as active in a plethora of cell types and tissues, however there is limited understanding about their relationship to overall gene function and the resulting gene-phenotype relationships. To gain insights into the mammalian regulatory landscape and its potential impact on phenotypic outcome, we focused our analysis on tissue-specific enhancers. By generating a catalogue of super, typical and weak enhancers in multiple mouse tissues we systematically investigated their roles in gene function. From multiple aspects such as gene expression, PPI networks and phenotypes, our study now provides evidence that $\mathrm{SE}$ and TE associated genes share common phenotypic outcomes even though their expression profiles and overall numbers in the genome differ.

SEs are comprised of dense enhancer clusters spanning large genomic regions and are associated with master transcription factors and other key cell identity genes $[21,31]$. We observed that compared to TEs, SEs consists of a large number of constituent enhancers, however, the mechanistic mode of action of these individual constituent elements is not well understood. It remains unclear whether the constituent enhancers exert an additive or a more complex cooperative effect on target gene expression. Using our genome-wide enhancer maps, we sought to examine the effect of constituent enhancer density on the total-expression of genes at a genome-wide scale. Our results show that globally, totalexpression levels of genes are weakly correlated with the number of constituent enhancers. The constituent enhancer density explains only a small fraction of the variation in gene expression, indicating a complex rather than a linear additive relationship between constituent enhancers and target gene expression. Not all constituent enhancers appear to contribute to the transcriptional output with the same strength, suggesting some constituent enhancers may make small contributions therefore helping to fine tune the expression patterns of their associated genes. This observation is consistent with previous in vivo experiments showing the effect of deleting individual SE constituents on target gene expression is highly variable $[50,69,70]$. SE constituents have more chromatin interactions among themselves [47], suggesting these constituent enhancers may have an effect on one another's contribution towards the target gene transcriptional activity. However, we cannot rule out the possibility that some constituent enhancers may have a redundant function in transcriptional activation [71]. It should be noted this study is a computational prediction and has limitations. In order to accurately calculate the impact of constituent enhancers on target gene expression, it is important to know which constituent enhancers are real and/or active alongside the gene(s) they regulate.

Prior research has thoroughly investigated the role of SEs in complex traits, showing that disease-causing SNPs are more enriched in SEs of disease-relevant cell types [21, 40,41]. However, little research has been conducted to systematically examine the effect of SEs and TEs on diseases. Here, we investigated the mammalian phenotype and disease associations of SE and TE associated genes. We identified that both the SEC and TEC are significantly enriched in phenotypes and diseases in 
the corresponding tissue-types (Fig. 3), emphasising that phenotypes are governed by tissue-specific enhancers. Using phenotyping data from knockout mouse lines of enhancer associated genes, we show that there is no significant difference in severity and breadth of phenotypes produced from knockouts of SEC and TEC (Fig. 4, Additional file 1: Figure S11), which underscores the importance of both enhancer classes in disease causation. In addition, no difference in enrichment of mouse essential genes and number of eQTL associations was identified among SEC and TEC. Overall, we did not find any significant contrast between the potential phenotypic impact of SEC and TEC, suggesting that functional testing of all enhancers irrespective of categories is fundamental in making any conclusions about their functional significance and phenotypic impact. Although the majority of key cell identity genes and TFs are associated with SEs, the 'peripheral' genes associated with TEs appear to equally contribute towards disease aetiology. A possible explanation to this surprising result is the existence of an 'omnigenic' architecture [72] where regulatory networks are densely inter-related such that TE associated genes expressed in disease relevant cell types can collectively impact the regulation of key cell identity genes. To this end, we hypothesised that tissue-specific enhancer associated genes are components of protein complexes involved in aberrant disease-causing biochemical processes and could be potential therapeutic targets. Our PPI analysis show that enhancer associated genes with no prior corresponding tissue-type phenotypic associations preferentially interact with known phenotypeassociated genes. This observation suggests that these enhancer associated genes could serve as novel targets for diseases.

Finally, using a machine learning approach, we systematically evaluated the capability of TSREs and other molecular properties to predict gene-phenotype associations in mouse (Fig. 7). By comparing classifiers trained on different gene features, we found the classifier with all the gene features combined performs the best to predict gene-phenotype associations. Our results also reveal that PPI data have a high predictive capacity to infer mammalian gene-phenotype associations, while regulatory data provides a modest but additive source of information. Further examination of the top scoring falsepositive predictions shows their promising application in generating hypothesis about gene function and in identification of potential novel disease targets. Such prediction models can assist in prioritising genes in mouse knockout and genome editing studies. They could also help in selecting the most relevant phenotyping procedures (which often involves costly assays) for transgenic mice models.

\section{Conclusion}

In this study, we systematically characterised different enhancer types with the goal of investigating their roles in gene function. We found that super- and typicalenhancers have different effect on gene expression, but both are preferentially associated with relevant tissuetype mammalian phenotypes and human diseases. We show that genes associated with super- and typicalenhancers exhibit no difference in phenotype effect size or pleiotropy suggesting they share common phenotypic outcomes. Our findings in a diverse range of mouse tissues present opportunities for molecular experiments to investigate regulatory mechanisms in mouse models of human diseases.

\section{Methods}

\section{Learning chromatin states and segmentation of the mouse genome}

First, the ChIP-Seq data for histone H3 lysine 4 monomethylation (H3K4me1), histone $\mathrm{H} 3$ lysine 4 trimethylation (H3K4me3) and histone H3 lysine 27 monoacetylation (H3K27ac) in 22 mouse tissues and cell lines were collected from ENCODE project (LICR lab) in the form of sequence alignments (BAM files mapped to $\mathrm{mm} 9$ mouse genome). The 22 epigenomes include 14 adult tissues: BAT (brown adipose tissue), bone marrow, cerebellum, cortex, heart, kidney, liver, lung, olfactory bulb, placenta, small intestine, spleen, testis and thymus; 2 embryonic tissues: limb and whole brain; and 6 cell lines: bone marrow derived macrophage, CH12 (B-cell lymphoma, GM12878 analog), Esb4 (mouse embryonic stem cells), Es-E14 (mouse embryonic stem cell line E14), MEF (mouse embryonic fibroblast), MEL (leukemia, K562 analog). Next, we used a multivariate hidden Markov model called ChromHMM to integrate all the ChIP-Seq data and summarise into easily illustratable annotations. The chromatin states were jointly learned across 22 mouse tissues using default parameters. Several HMM models were produced consisting of 4-8 chromatin states and identified the 6 state model to provide sufficient resolution to isolate biologically meaningful chromatin states. The resulting chromatin states were then annotated based on the biological significance of the frequencies of combined histone marks. Using this approach, potential active promoter $(404,016)$, weak promoter $(647,185)$, strong enhancer $(1,075,608)$ and weak enhancer $(2,068,844)$ annotations were mapped across 22 mouse tissues and cell types. To validate our predicted promoter states (states 1 and 2), we compared 217,678 unique non-overlapping promoters to 22,707 known protein coding genes ( $\mathrm{mm} 9$ ensembl genes v67; $10 \mathrm{~kb}$ upstream, $100 \mathrm{bp}$ downstream of TSS) and recovered $81.66 \%$ of known promoters. Similarly, to validate the strong enhancer predictions (state 4), we compared 386,222 unique non-overlapping enhancers to 363 experimentally validated VISTA mouse enhancers and recovered 
91.18\% of VISTA enhancers from our predictions. Chromatin states with $<0.95$ posterior probability were filtered resulting in 923,791 strong enhancer (state 4); 309,581 active promoter (state 2); 2,531,993 weak enhancer (state 6); and 427,251 weak promoter (state 1) high confidence annotations respectively.

\section{Identifying tissue-specific regulatory elements}

To identify tissue-specific regulatory regions across the 22 tissues, we implemented the Tau method which has been previously used to detect tissue-specific expression $[43,44]$. Tau is a measure of tissue specificity index which takes into account number of tissues and normalised expression in each tissue and outputs a score for each gene. To implement this method, we constructed matrices of chromatin state posterior probabilities for strong enhancers, active promoters, weak enhancers and weak promoters with dimension $n \times s$, where $n$ is the number of regulatory elements and $s$ is the number of tissues (i.e. 22). Each row of the matrix is a genomic location of the regulatory element (200 bp in length) and columns represents its posterior probability across all the tissues. The matrices were filtered such that only the regulatory elements with a posterior probability $\geq 0.95$ in at least one tissue were retained. The Tau score for each regulatory element was calculated by the following equation:

$$
\tau_{r e g}=\frac{\sum_{i=1}^{N}\left(1-\widehat{x_{i}}\right)}{N-1} ; \widehat{x_{i}}=\frac{x_{i}}{\max \left(x_{i}\right)}
$$

where $N$ is the number of tissues and $x_{i}$ is the posterior probability value. Using the thresholds suggested in [43], the regulatory elements were categorised into low $\left(\tau_{\text {reg }} \leq 0.15\right)$, intermediate $\left(0.15<\tau_{\text {reg }}<0.85\right)$, high $\left(0.85 \leq \tau_{\text {reg }}<1\right)$ and absolute tissue-specific $\left(\tau_{\text {reg }}=1\right)$.

\section{Correlating TSREs with DNasel hypersensitive sites}

For DNasel accessible regions, we collected DNasel hypersensitivity sites (DHS) in 11 tissues (Cerebellum, CH12, Es-E14, Heart, Kidney, Liver, Lung, MEL, Spleen, Thymus, Wbrain) from ENCODE (UW lab) in the form of hotspots. The mean of DNaseI signal was computed wherever multiple replicates were available within ENCODE. The genomic coordinates of tissue-specific enhancer and promoter elements were compared with DNaseI hypersensitive hotspots using BEDTools [73] and the DNaseI signal in each tissue or cell line was extracted. We restricted the extraction of DNaseI signal to cases where $100 \%$ of the enhancer or promoter region overlapped the DHS hotspot, otherwise no DNaseI activity was assumed and a value of " 0 " was assigned to that enhancer or promoter. This resulted in a matrix of
DNaseI signal corresponding to the posterior probability matrix of tissue-specific enhancers and promoters. To quantify the concordance between TSREs (tissue-specific enhancers and promoters) and DHS, Pearson's correlation between posterior probability of their respective chromatin state and the corresponding DNasel signal was calculated. The pairwise correlations between the tissues were visualised in a heatmap and rows and columns were ordered based on hierarchical clustering (Additional file 1: Figure S2b and S2c).

\section{Distinguishing super-enhancers from typical-enhancers}

To identify SEs in mouse, we implemented an approach similar to previously used by [31]. Using the ROSE algorithm, tissue-specific enhancers within a distance of $12.5 \mathrm{~kb}$ were stitched together into cohesive units and ranked based on their H3K27ac signal. A TSS exclusion size of $2000 \mathrm{bp}$ was used to exclude tissue-specific enhancers within $\pm 2 \mathrm{~kb}$ of a known TSS to remove any promoter bias. The algorithm calculates a threshold of the inflection point for H3K27ac signal. The stitched cohesive units with H3K27ac signal higher than the estimated threshold are defined as SEs while the remaining cohesive units are termed as TEs.

The metagene profiles of mean H3K27ac signal across all the SEs and TEs (Fig. 1d, Additional file 1: Figure S3) were generated using ngs.plot [74]. Metagene plots are centered on the enhancers and display average ChIP-Seq read density over all the enhancer regions and surrounding windows of $2 \mathrm{~kb}$. For visual comparison between profiles of SEs and TEs in a tissue, the range of the $y$ axis were synchronised. For comparing the H3K4me1, H3K27ac and DNAseI hypersensitivity signal over the stitched enhancers (Additional file 1: Figure S4), the read density over these regions was calculated in reads per million (rpm). For H3K4me1 and H3K27ac ChIP-Seq signal, the input control density was subtracted in rpm. The read density for each feature was then normalized by dividing the signal at each enhancer by the maximum signal in each feature. The stitched enhancers for each feature on $\mathrm{x}$-axis are ranked according to the H3K27ac ChIP-Seq signal.

\section{Effect size calculation}

The non-parametric effect size (ES) was calculated as the difference in medians of the two groups divided by the pooled median absolute deviation (MAD). The following formula was used:

$$
E S=\frac{\text { Median }_{1}-\text { Median }_{2}}{M A D_{\text {pooled }}} ; M A D_{\text {pooled }}=\sqrt{\frac{M A D_{1}^{2}+M A D_{2}^{2}}{2}}
$$




\section{Associating TSREs to potential target genes}

We used GREAT [48] to associate tissue-specific regulatory elements to potential target genes in each tissue. In cases where GREAT predicted multiple target genes for a particular TSRE, the nearest gene was selected as the primary predicted target for all further downstream analysis. GREAT was run using default parameters on $\mathrm{mm} 9$ assembly and the whole genome was selected for control background regions. The coordinates of TSREs and their associated genes in all tissues are provided in Additional file 2. To examine the consistency of our enhancer-gene assignments with other datasets, we compared them to previously reported topologically associated domains (TADs) [49] and enhancer-promoter units (EPUs) [6] in mouse. The enhancer-gene pairs across the 22 tissues were merged together for this comparison. The TADs (in mESC and cortex) were compared to the enhancergene pairs to examine if the enhancer-gene pair overlaps the same TAD (Additional file 3). Only the cases where both enhancer and its associated gene overlapped a TAD were used. We identified 96.62 and $93.57 \%$ of our enhancer-gene pairs to be in the same TADs annotated in cortex and mESC respectively. A similar comparison was done with EPUs which revealed $87.23 \%$ of our predicted enhancer-gene pairs to be in the same EPU.

\section{Expression analysis of enhancer associated genes}

For investigating the expression of enhancer associated genes, RNA-Seq data for all 22 tissues and cell lines was collected from ENCODE as read alignments (BAM files). Data for cell lines $\mathrm{CH} 12$ and Es-E14 was collected from Standford/Yale lab while rest of the data was retrieved from LICR lab. From the BAM files, the read counts over all genes ( $\mathrm{mm} 9$, ensembl v67) were quantified using HTSeq [75] and expression of each gene was calculated in RPKM (Reads Per Kilobase of transcript per Million mapped reads) in each tissue/cell line. A mean RPKM value was calculated for multiple biological replicates from ENCODE.

To examine the relationship between enhancers and expression of their target genes, data from all 22 tissues was combined into gene-tissue pairs and grouped into three classes based on their enhancer association: (1) gene-tissue pairs associated with SEs (SEC); (2) genetissue pairs associated with TEs (TEC); and (3) genetissue pairs associated with weak/poised enhancers (WEC). In order to quantify tissue-specific expression of target genes, we calculated the tissue specificity index for each gene using the Tau method described earlier. We constructed a matrix of expression values with dimensions $t \times s$, where $t$ is the total number of genes and $s$ is the number of tissues/cell lines. Genes not expressed in any tissue were deleted from the matrix leaving genes expressed in at least 1 tissue. The RPKM values were $\log 2$ transformed and quantile normalised (QN) (using the normalize.quantiles function in preprocessCore $R$ package) to allow easier comparison of gene expression across tissues. Genes were then sorted by ascending QN value and divided into deciles of equal density and placed into 10 bins. The lowest decile (lowest 10\% of genes by QN value) was placed in bin 1, the next lowest was placed in bin 2, and so on until the top $10 \%$ of QN values were placed in bin 10 . The Tau value $\left(\tau_{\text {exp }}\right)$ for each gene was calculated as:

$$
\tau_{\text {exp }}=\frac{\sum_{i=1}^{N}\left(1-\widehat{y}_{i}\right)}{N-1} ; \widehat{y}_{i}=\frac{y_{i}}{\max \left(y_{i}\right)}
$$

where $N$ is the total number of tissues, $y_{i}$ is the normalised expression bin profile component of the gene in tissue $i$. In order to associate $\tau_{\text {exp }}$ values to tissues, Taufraction $\left(\tau_{\text {exp - frac }}\right)$ for each gene in every tissue was calculated as $\frac{\operatorname{texp}_{\operatorname{la}} r_{i}}{M}$ where $r_{i}$ is the expression of the gene (in RPKM) in tissue $i$ and $M$ is the maximum expression of the gene (in RPKM) across all the tissues. Based on $\tau_{\text {exp-frac }}$ score, the genes were categorised into low ( $\tau_{\text {exp }}$ - frac $\leq 0.20)$, intermediate $\left(0.20<\tau_{\text {exp }- \text { frac }}<0.85\right)$ or high $\left(\tau_{\text {reg }} \geq 0.85\right)$ tissue-specific expression in the corresponding tissues. Housekeeping genes were identified based on a strict $\tau_{\exp }$ threshold. Genes with low $\tau_{\exp }$ score $(\leq 0.20)$ are uniformly expressed across all the tissues and were considered to be housekeeping genes. We identified 1252 housekeeping genes using this threshold out of which 1171 were protein-coding genes.

To visualise the distinct number of enhancer tissuetypes calculated for each enhancer-associated gene (Fig. 2f), we generated two binary matrices for SEC and TEC in 22 tissues. The rows in the matrix represented enhancer associated genes and columns represented different tissues. A value of " 1 " or " 0 " was assigned to the cells in the matrix depending on if the gene was identified to be associated with the enhancer of that tissue or not respectively. The heatmaps in Fig. $2 \mathrm{f}$ were first sorted on the number of enhancer tissue-types and then sorted by the order of tissues across the columns.

\section{GO, mammalian phenotype and disease enrichment analysis}

To investigate the molecular functions and biological processes linked with enhancer associated genes, we combined the SE and TE associated genes across the 22 tissues to make two unique lists. This resulted in 3617 genes to be only associated with SEs and 11,437 genes to be only associated with TEs. These gene sets were then used for GO enrichment analysis using ToppGene suite [76] (Additional file 7). The enrichment of mammalian phenotypes and human diseases was calculated 
individually in each tissue using the ToppFun tool in ToppGene suite. The enrichment of housekeeping genes among SEC and TEC was calculated using Fisher's exact test. For background, total number of protein coding genes in our annotation set was used. SEC is significantly depleted for housekeeping genes (155/3617; $p=$ 0.012 , $\mathrm{OR}=0.82)$ while TEC is enriched $(686 / 11,437$; $p=2.7 \times 10^{-11}, \mathrm{OR}=1.49$ ).

\section{Mouse gene knockout data}

The mouse phenotyping data of enhancer associated gene knockouts was extracted from IMPC (International Mouse Phenotyping Consortium). All the statistically significant genotype-phenotype associations and their phenotyping data in IMPC release version 5.0 were used. This version compromised of phenotype data for 3323 gene knockouts, with 2900 genes significantly associated with at least one phenotype attribute $\left(p<10^{-4}\right)$. To quantify the severity of phenotypes, we used the percentage change value from each procedure. The percentage change is normalised effect size, which is scaled to make it comparable across various procedures and parameters [77]. The percentage change between SE and TE associated genes was compared for several standardised phenotyping procedures. The phenotype procedure protocols are described in IMPReSS (https://www.mousephenotype.org/impress). All the parameters within a procedure were grouped together for this analysis. For computing the enrichment of mouse essential genes in SEC and TEC, genes producing a lethal homozygous knockout (960 genes out of 2900) were used.

\section{GTEx expression quantitative trait loci}

The official set of GTEx v8 significant variant-gene associations based on permutations and conditionally independent eQTLs mapped using stepwise regression were used for the analysis. For each gene in SEC and TEC, we extracted and counted the total number of eQTL associations. This analysis was performed in the following tissues: cerebellum, cortex, heart, liver, lung, small intestine, spleen and testis.

\section{Known gene-phenotype associations}

All the gene-phenotype associations in mouse were extracted from MGD. The Mouse Phenotypic Alleles report (MGI_PhenotypicAllele.rpt) was collected from MGD on 14th June 2017.

\section{Protein-protein interaction maps}

The predicted protein-protein interactions among the genes of interest were extracted from the STRING database [58] using the $\mathrm{R}$ package STRINGdb. A score threshold of 900 was implemented to extract potential interactions with the highest confidence and reduce false-positives. These interaction maps were visualised as networks using the iGraph package in R. The known gene-phenotype associations (from MGD) in the network were labelled as "known" while the remaining genes were marked as "novel". A permutation test was performed to identify if the observed number of interactions between known and novel genes are more than what we would expect by random (Additional file 1: Figure S14). We added randomly selected protein-coding genes equal to the number of genes known to be associated with phenotypes in the network and extracted their interactions from STRING. The number of interactions (edges) between randomly added genes and known phenotype genes were then counted. This was repeated 1000 times to produce a distribution of expected number of edges and the $p$-value was calculated as $p=y / N$, where $y$ is number of permuted random-known edges greater than the observed novel-known edges and $N$ is the total number of items in our distribution (i.e. 1001).

\section{Cistrome data}

For the analysis of transcription factor binding sites colocalised with different enhancer sets, we used a cell type independent cistrome, the general genomic map of regions bound by particular TFs in any cell type [78]. The cistrome is based on uniformly reprocessed ChIPSeq data from the GTRD database [79] across all the cell types and conditions. The cistrome regions are classified into four reproducibility categories (A,B,C,D): A - regions supported by ChIP-Seq data from two different experimental data sets (at least one was accompanied by control data) and different ChIP-Seq peak calling tools; B - regions supported by peak calls from two different experimental data sets (at least one was accompanied by control data); C - regions supported by peak calls from a single experimental data set with control data and different peak calling tools; D - all other reproducible regions (supported by more than one peak). A and B categories were taken into the analysis by default. For TFs with a limited number of ChIP-Seq data sets, we added regions from $C$ and $D$ categories when it was necessary to get at least 100 peaks. As an additional filter for cistrome, we used TF binding motifs from HOCOMOCO to annotate motif occurrences in cistrome regions with SPRYSARUS [80] using the default motif $p$-value threshold of $5 \times 10^{-4}[81]$ and then discarded cistrome segments without motif occurrences.

\section{Enrichment of TFBS in SEs and TEs}

To calculate the enrichment of TF binding within SE and TE constituents, we first merged the neighbouring constituent enhancers within $400 \mathrm{bp}$ into prolonged extended enhancer segments in each tissue. These 
extended enhancer segments were then used to generate the control regions; more precisely, for each enhancer segment of length $\mathrm{L}$, we located two segments (enhancer shades) of length $\mathrm{L}$, one at $100 \mathrm{~L}$ upstream and the other at $100 \mathrm{~L}$ downstream. This produced a set of control segments of the same lengths and similar global genomic context as the enhancer segment under study. We checked if any control segments overlapped other constituent enhancers, but such cases contributed only 1$2 \%$ of the total number of control regions and were safely ignored. The extended enhancer segments and control regions were then intersected with the cistrome peaks of each TF and split into two groups; overlapping (if least $1 \mathrm{bp}$ overlapped) and non-overlapping with the cistrome. The Fisher's exact test on $2 \times 2$ contingency tables was used to assess the statistical significance of TF cistrome peaks overlapping constituent enhancers (SE or $\mathrm{TE}$ ) versus control regions (Additional file 8). The resulting $p$-values were corrected for multiple testing using Bonferroni correction. Note that the cistrome segments of a TF could significantly colocalise with enhancers in several different cell types, therefore, we counted the number of significant enrichments as TFtissue pairs. We also performed the analysis with only the cistrome segments that contain high scoring motif hits from HOCOMOCO. The results were very similar to the analysis where all cistrome segments were considered; about $10 \%$ of TFs did not have known binding motifs, and for TFs with known motifs, about 90\% of significant TF-tissue pairs were independent from whether the motifs were considered or not (Additional file 8).

\section{TFBS density analysis}

To calculate the TFBS density of each TF, we intersected each enhancer element with the TF cistrome peaks. Within these overlapping regions, we predicted the binding motif occurrences of the corresponding TF using HOCOMOCO-v11 motifs. In cases where HOCOMOCO contained multiple motif models for a single TF, all motifs were used and the binding sites exceeding the cistrome $p$-value threshold of 0.0005 were retained. Density was calculated as the total genomic coverage of motifs (in bp) divided by the total coverage of enhancercistrome intersection (in bp). We calculated densities for only those enhancers (constituent enhancers of SEs or TEs) which had at least one motif occurrence in its intersection with the cistrome. The Wilcoxon Rank Sum Test was then used to compare the TFBS densities of TF-tissue pairs in SEs and TEs (each TF-tissue pair was compared individually between SEs and TEs). The noncorrected $p$-values were used to order the TF-tissue pairs by their level of TFBS density disparity between SEs and TEs. The TF-tissue pairs were grouped into bins based on their $p$-value and the number of TF-tissue cases where its TFBS density was more in SEs compared to TEs, or vice versa, were counted (Additional file 1: Figure S15b).

\section{Predicting gene-phenotype associations}

To predict mammalian gene-phenotype associations, features were extracted from TSREs, expression, transcription factor binding and PPI data for all protein-coding genes. From the TSRE profiles across 22 tissues, strongenhancers and active promoters associated with each protein-coding gene were extracted. A score representing the tissue-specific enhancers and promoters in each tissue was computed as $S_{g t}=\sum_{i=1}^{N}(P)$, where $S_{g t}$ is the score of gene $g$ in tissue $t$; $N$ is the total number of strong enhancer or active promoter elements associated with gene $g$ in tissue $t$; and $P_{i}$ is the posterior probability of the associated strong enhancer or active promoter element emitted by the ChromHMM model. The RPKM values for each gene, quantified using ENCODE's RNASeq data in 22 tissues were used as a feature for expression data. For TF binding associated with each gene, we first we selected all cistrome regions overlapping -500 bp and +100 bp of TSS (for each gene, we considered all transcripts from gencode vM15). Then, we calculated the $-\log 10(p$-value) of HOCOMOCO motif hits within these cistrome regions (aggregating over all motifs if there were multiple models for a particular TFBS). The respective values for each TF were taken as the TFBS features. The final set of the TFBS features covered all TFs for which we had the ChIP-Seq cistrome peaks and a binding motif model $(n=297)$. For PPIs, all the protein interactions in mouse were collected from STRING database version 10.5. For a gene $g$, its PPI connectivity with all strong enhancer and active promoter associated genes in tissue $t$ was calculated as $P P I_{g t}=\sum_{i=1}^{N}(I)$, where $N$ is the total number of enhancer or promoter associated genes in tissue $t$ and $I_{i}$ is the combined interaction score between gene $g$ and $i^{\text {th }}$ gene. Similarly for each gene, its PPI connectivity with all genes known to be associated with the phenotype domain to be predicted was computed as $P P I_{g-p h e n}=\sum_{i=1}^{M}(I)$, where $I$ is the interaction score and $M$ is the total number of known phenotype associated genes from MGD.

The random forest classifier was implemented in $R$ using randomForest and caret package [82]. We sought to predict gene-phenotype associations from 13 different phenotypes relevant to at least one tissue type in our dataset. The known gene-phenotype associations from 
MGD (top level MP annotations) served as truepositives for the classifier models. The random forest classifier was trained on a subset of genes (using default parameters), where features described above were used as predictor variables and phenotype calls from MGD as the response variable. This model was used to predict gene-phenotype associations in the remaining set of genes not used in the training of the model. The preProcess function in caret was used to centre and scale all the gene features. Down-sampling was employed on the training data to avoid the impact of class imbalance on model fitting. Model optimisation across these parameters was performed using $\mathrm{k}$-fold cross validation technique, to choose the model with the best ROC (parameters used: method $=$ "repeatedcv", number $=5$, repeats $=5$, metric $=$ "ROC"). In order to compare the predictive capability of various gene features, 10 different models with different gene feature combinations were built for each phenotype domain (130 models in total). Each of these classifier was assessed by generating ROC and PR curves based on 5-fold cross validation repeated 10 times. The cross validation results were then averaged for comparison and reporting purposes. The top false positives hits (prediction probability $\geq 0.90$ ) were examined using the Open Target Platform and the DisGeNET discovery platform to validate the novel predictions. Predictions from only those phenotype domains were investigated which had a corresponding disease class in Open Targets and DisGeNET platform. As a result, predictions from 12 phenotypes were examined with Open Targets platform and predictions from 9 phenotypes were examined with DisGeNET (see Additional file 10).

\section{Supplementary information}

Supplementary information accompanies this paper at https://doi.org/10. 1186/s12864-020-07109-5.

Additional file 1: Figure S1. Chromatin state segmentation and characterisation across 22 mouse tissues. Figure S2. Overview of tissuespecific regulatory elements in the mouse genome. Figure S3. H3K27ac activity within SES and TEs. Figure S4. Enrichment of chromatin marks over stitched cohesive enhancer units. Figure S5. Chromatin activity within SE and TE constituent enhancers. Figure S6. Region-gene associations of regulatory elements. Figure S7. Relationship between enhancer activity and their target gene expression. Figure S8. Impact of constituent enhancer density on target gene expression. Figure S9. Enhancer usage switch associated with genes within SEC and TEC with multiple enhancer tissue-types. Figure S10. Genomic view of genes demonstrating enhancer usage switch. Figure S11. Breadth of phenotypes associated with SE and TE gene knockouts in mouse. Figure S12. Number of eQTLS associated with genes within SEC and TEC. Figure S13. Protein-protein interaction maps of enhancer associated genes. Figure S14. Proteinprotein interaction simulations. Figure $\mathbf{S 1 5}$. Transcription factor binding within SE and TE constituents. Figure S16. Performance of random forest classifiers to predict mammalian gene-phenotype associations. Figure S17. Precision and recall of classifiers used to predict gene-phenotype associations. Figure S18. Evaluation of top-scoring false-positives using the Open Targets platform. Table S1. Mammalian phenotype and human disease ontology terms enriched in genes associated with weak-enhancers.
Additional file 2. List of genes associated with SES (SEC) and TEs (TEC) in 22 tissues.

Additional file 3. Comparison of enhancer-gene pairs with TADs and EPUs.

Additional file 4. Enhancer tissue-type association of SEC and TEC. Additional file 5. Enhancer usage switch scores of genes within SEC and TEC.

Additional file 6. Gene Ontology enrichment of genes within SEC and TEC.

Additional file 7. Mouse phenotype and human disease enrichment of genes within SEC and TEC in 22 tissues.

Additional file 8. Enrichment of TF cistrome peaks within SE and TE regions.

Additional file 9. Performance metrics of all random forest classifiers.

Additional file 10. Exploration of top scoring predictions and the evidence supporting their association with the corresponding diseases.

\section{Abbreviations}

TFs: Transcription factors; DHS: DNasel hypersensitive sites;

IMPC: International mouse phenotyping consortium; SEs: Super-enhancers; TEs: Typical-enhancers; TSREs: Tissue-specific regulatory elements;

TADs: Topological associated domains; EPUs: Enhancer-promoter units; TSS: Transcription start site; SEC: Super-enhancer class; TEC: Typical-enhancer class; WEC: Weak-enhancer class; ES: Effect size; GO: Gene ontology; OR: Odds ratio; PPI: Protein-protein interactions; TFBS: Transcription factor binding site; ROC: Receiver operating characteristic; PR: Precison-recall; AUC: Area under the curve

\section{Acknowledgements}

Not applicable.

\section{Authors' contributions}

S.S. conducted most of the research and data analysis. I.E.V., I.V.K., V.J.M. prepared the cistrome data and performed all the TF enrichment and density analysis. I.E.V. analysed and generated TF binding features for the classifier. I.V.K., S.G., J.W. provided help on machine learning and statistics. M.M.S. and A.-M.M. jointly supervised this work, provided critical feedback and helped shaped the research and analysis. S.S. wrote the manuscript with help from M.M.S. and A.-M.M. All authors contributed, read, and approved the final version

\section{Funding}

Research reported in this publication was supported by the Medical Research Council (MC_U142684171) and National Human Genome Research Institute of the National Institutes of health (UM1HG006370). The ChIP-

Seq cistrome and motif analysis was supported by RSF [grant 20-74-10075 to I.V.K.]. I.E.V. was personally supported by the Skoltech Systems Biology Fellowship. The content is solely the responsibility of the authors and does not necessarily represent the official views of the National Institutes of Health.

\section{Availability of data and materials}

The datasets supporting the conclusions of this article are included within the article and its additional files. Source code of the analysis can be found here: https://github.com/MRC-Harwell/SuperEnhancers

Ethics approval and consent to participate

Not applicable.

\section{Consent for publication}

Not applicable.

\section{Competing interests}

The authors declare no competing interests.

\section{Author details}

${ }^{1}$ Mammalian Genetics Unit, MRC Harwell Institute, Oxfordshire OX11 ORD, UK. ${ }^{2}$ Vavilov Institute of General Genetics, Russian Academy of Sciences, Gubkina 3, Moscow 119991, Russia. Institute of Protein Research, Russian Academy of 
Sciences, Institutskaya 4, Pushchino, Moscow Region 142290, Russia. ${ }^{4}$ Engelhardt Institute of Molecular Biology, Russian Academy of Sciences, Vavilova 32, Moscow 119991, Russia. Institute of Translational Medicine, University Hospitals Birmingham NHS Foundation Trust, Birmingham B15 2TH, UK. ${ }^{6}$ Institute of Cancer and Genomic Sciences, University of Birmingham, Birmingham B15 2TT, UK. ${ }^{7}$ Moscow Institute of Physics and Technology, 9 Institutskiy per., Dolgoprudny, Moscow Region 141700, Russia.

\section{Received: 12 November 2019 Accepted: 29 September 2020}

Published online: 02 November 2020

\section{References}

1. Lee TI, Young RA. Transcriptional regulation and its Misregulation in disease. Cell. 2013;152(6):1237-51.

2. ENCODE Project Consortium, T. An integrated encyclopedia of DNA elements in the human genome. Nature. 2012;489:57.

3. FANTOM Consortium, T., t.R. PMI, and CLST. A promoter-level mammalian expression atlas. Nature. 2014;507:462.

4. Bernstein BE, et al. The NIH Roadmap Epigenomics mapping Consortium. Nat Biotechnol. 2010;28(10):1045-8.

5. Heintzman ND, et al. Distinct and predictive chromatin signatures of transcriptional promoters and enhancers in the human genome. Nat Genet. 2007;39(3):311-8.

6. Shen $Y$, et al. A map of the cis-regulatory sequences in the mouse genome Nature. 2012;488(7409):116-20.

7. Hoffman MM, et al. Integrative annotation of chromatin elements from ENCODE data. Nucleic Acids Res. 2013;41(2):827-41.

8. Roadmap Epigenomics Consortium, T, et al. Integrative analysis of 111 reference human epigenomes. Nature. 2015;518:317.

9. Creyghton MP, et al. Histone H3K27ac separates active from poised enhancers and predicts developmental state. Proc Natl Acad Sci U S A. 2010;107(50):21931-6.

10. Kim $\mathrm{TH}$, et al. A high-resolution map of active promoters in the human genome. Nature. 2005;436:876.

11. Maston GA, Evans SK, Green MR. Transcriptional regulatory elements in the human genome. Annu Rev Genomics Hum Genet. 2006;7:29-59.

12. Schoenfelder S, Clay I, Fraser P. The transcriptional interactome: gene expression in 3D. Curr Opin Genet Dev. 2010;20(2):127-33.

13. Gibcus JH, Dekker J. The Hierarchy of the 3D Genome. Mol Cell. 2013;49(5): 773-82.

14. Sanyal A, et al. The long-range interaction landscape of gene promoters. Nature. 2012;489(7414):109-13.

15. Kagey $\mathrm{MH}$, et al. Mediator and cohesin connect gene expression and chromatin architecture. Nature. 2010:467(7314):430-5.

16. Liu W, et al. Brd4 and JMJD6-associated anti-pause enhancers in regulation of transcriptional pause release. Cell. 2013;155(7):1581-95.

17. Kleinjan DA, Lettice LA. Long-range gene control and genetic disease. Adv Genet. 2008:61:339-88.

18. Bhatia S, Kleinjan DA. Disruption of long-range gene regulation in human genetic disease. Hum Genet. 2014;133(7):815-45.

19. Hindorff $L A$, et al. Potential etiologic and functional implications of genome-wide association loci for human diseases and traits. Proc Natl Acad Sci U S A. 2009;106(23):9362-7.

20. Maurano MT, et al. Systematic localization of common disease-associated variation in regulatory DNA. Science. 2012;337(6099):1190-5.

21. Hnisz D, et al. Super-enhancers in the control of cell identity and disease. Cell. 2013:155(4):934-47.

22. Brown SDM, Moore MW. Towards an encyclopaedia of mammalian gene function: the international mouse Phenotyping Consortium. Dis Models Mech. 2012;5(3):289-92.

23. Ring $\mathrm{N}$, et al. A mouse informatics platform for phenotypic and translational discovery. Mamm Genome. 2015;26(9-10):413-21.

24. Dickel DE, et al. Ultraconserved Enhancers Are Required for Normal Development. Cell. 2018:172(3):491-9 e15.

25. Li $Y$, et al. CRISPR reveals a distal super-enhancer required for Sox2 expression in mouse embryonic stem cells. PLoS One. 2014;9(12):e114485.

26. Seruggia D, et al. Functional validation of mouse tyrosinase non-coding regulatory DNA elements by CRISPR-Cas9-mediated mutagenesis. Nucleic Acids Res. 2015;43(10):4855-67

27. Dao LTM, et al. Genome-wide characterization of mammalian promoters with distal enhancer functions. Nat Genet. 2017;49(7):1073-81.
28. Canver MC, et al. BCL11A enhancer dissection by Cas9-mediated in situ saturating mutagenesis. Nature. 2015:527:192.

29. Moorthy SD, Mitchell JA. Generating CRISPR/Cas9 mediated Monoallelic deletions to study enhancer function in mouse embryonic stem cells. J Vis Exp. 2016;110:e53552.

30. Cunningham TJ, et al. Genomic knockout of two presumed forelimb Tbx5 enhancers reveals they are nonessential for limb development. Cell Rep. 2018;23(11):3146-51.

31. Whyte WA, et al. Master Transcription Factors and Mediator Establish SuperEnhancers at Key Cell Identity Genes. Cell. 2013;153(2):307-19.

32. Loven J, et al. Selective inhibition of tumor oncogenes by disruption of super-enhancers. Cell. 2013;153(2):320-34.

33. Pelish $\mathrm{HE}$, et al. Mediator kinase inhibition further activates super-enhancerassociated genes in AML. Nature. 2015;526:273.

34. Huang J, et al. Dynamic control of enhancer repertoires drives lineage and stage-specific transcription during hematopoiesis. Dev Cell. 2016;36(1):9-23.

35. Adam RC, et al. Pioneer factors govern super-enhancer dynamics in stem cell plasticity and lineage choice. Nature. 2015;521(7552):366-70.

36. Fang $Z$, et al. Transcription factor co-occupied regions in the murine genome constitute T-helper-cell subtype-specific enhancers. Eur J Immunol. 2015:45(11):3150-7.

37. Ohba $\mathrm{S}$, et al. Distinct transcriptional programs underlie Sox9 regulation of the mammalian chondrocyte. Cell Rep. 2015:12(2):229-43.

38. Siersbæk $R$, et al. Transcription factor Cooperativity in early Adipogenic hotspots and super-enhancers. Cell Rep. 2014;7(5):1443-55.

39. Vahedi $\mathrm{G}$, et al. Super-enhancers delineate disease-associated regulatory nodes in T cells. Nature. 2015;520(7548):558-62.

40. Farh KK, et al. Genetic and epigenetic fine mapping of causal autoimmune disease variants. Nature. 2015;518(7539):337-43.

41. Parker SC, et al. Chromatin stretch enhancer states drive cell-specific gene regulation and harbor human disease risk variants. Proc Natl Acad Sci U S A. 2013;110(44):17921-6

42. Ernst J, Kellis M. ChromHMM: automating chromatin-state discovery and characterization. Nat Methods. 2012:9(3):215-6.

43. Yanai I, et al. Genome-wide midrange transcription profiles reveal expression level relationships in human tissue specification. Bioinformatics. 2005;21(5):650-9.

44. Kryuchkova-Mostacci N, Robinson-Rechavi M. A benchmark of gene expression tissue-specificity metrics. Brief Bioinform. 2017;18(2):205-14.

45. Beagrie RA, Pombo A. Gene activation by metazoan enhancers: diverse mechanisms stimulate distinct steps of transcription. Bioessays. 2016;38(9): 881-93.

46. Li W, et al. Functional roles of enhancer RNAs for oestrogen-dependent transcriptional activation. Nature. 2013:498(7455):516-20.

47. Dowen $\mathrm{JM}$, et al. Control of cell identity genes occurs in insulated neighborhoods in mammalian chromosomes. Cell. 2014;159(2):374-87.

48. McLean CY, et al. GREAT improves functional interpretation of cis-regulatory regions. Nat Biotechnol. 2010;28(5):495-501.

49. Dixon JR, et al. Topological domains in mammalian genomes identified by analysis of chromatin interactions. Nature. 2012:485(7398):376-80.

50. Shin HY, et al. Hierarchy within the mammary STAT5-driven Wap superenhancer. Nat Genet. 2016:48(8):904-11.

51. Cannon B, Nedergaard J. Brown adipose tissue: function and physiological significance. Physiol Rev. 2004;84(1):277-359.

52. Akazawa $\mathrm{H}$, Komuro I. Roles of cardiac transcription factors in cardiac hypertrophy. Circ Res. 2003;92(10):1079.

53. Martin C, et al. Chemokines acting via CXCR2 and CXCR4 control the release of neutrophils from the bone marrow and their return following senescence. Immunity. 2003;19(4):583-93.

54. Kim KK, Adelstein RS, Kawamoto S. Identification of neuronal nuclei (NeuN) as Fox-3, a new member of the Fox-1 gene family of splicing factors. J Biol Chem. 2009;284(45):31052-61.

55. Kim KK, et al. Rbfox3-regulated alternative splicing of numb promotes neuronal differentiation during development. J Cell Biol. 2013;200(4): 443-58.

56. Mansour MR, et al. Oncogene regulation. An oncogenic super-enhancer formed through somatic mutation of a noncoding intergenic element. Science. 2014;346(6215):1373-7

57. Dickinson ME, et al. High-throughput discovery of novel developmental phenotypes. Nature. 2016;537(7621):508-14. 
58. Franceschini A, et al. STRING v9.1: protein-protein interaction networks, with increased coverage and integration. Nucleic Acids Res. 2013;41(Database issue):D808-15.

59. Blake JA, et al. The mouse genome database (MGD): premier model organism resource for mammalian genomics and genetics. Nucleic Acids Res. 2011;39(Database issue):D842-8.

60. Ong $C T$, Corces VG. Enhancer function: new insights into the regulation of tissue-specific gene expression. Nat Rev Genet. 2011;12(4):283-93.

61. Scott EW, et al. Requirement of transcription factor PU.1 in the development of multiple hematopoietic lineages. Science. 1994;265(5178): 1573.

62. Pikkarainen S, et al. GATA transcription factors in the developing and adult heart. Cardiovasc Res. 2004;63(2):196-207.

63. Raymond CS, et al. Dmrt1, a gene related to worm and fly sexual regulators, is required for mammalian testis differentiation. Genes Dev. 2000;14(20): 2587-95.

64. Tasan $\mathrm{M}$, et al. An en masse phenotype and function prediction system for Mus musculus. Genome Biol. 2008;9(Suppl 1):S8.

65. Pena-Castillo $L$, et al. A critical assessment of Mus musculus gene function prediction using integrated genomic evidence. Genome Biol. 2008;9(Suppl 1):S2.

66. Yuan $Y$, et al. Predicting the lethal phenotype of the knockout mouse by integrating comprehensive genomic data. Bioinformatics. 2012;28(9):1246-52.

67. Koscielny $\mathrm{G}$, et al. Open targets: a platform for therapeutic target identification and validation. Nucleic Acids Res. 2017;45(Database issue): D985-94.

68. Pinero J, et al. DisGeNET: a comprehensive platform integrating information on human disease-associated genes and variants. Nucleic Acids Res. 2017: 45(D1):D833-d839.

69. Suzuki HI, Young RA, Sharp PA. Super-Enhancer-Mediated RNA Processing Revealed by Integrative MicroRNA Network Analysis. Cell. 2017;168(6):100014 e15.

70. Hay D, et al. Genetic dissection of the alpha-globin super-enhancer in vivo. Nat Genet. 2016;48(8):895-903.

71. Moorthy SD, et al. Enhancers and super-enhancers have an equivalent regulatory role in embryonic stem cells through regulation of single or multiple genes. Genome Res. 2017:27(2):246-58

72. Boyle EA, Li YI, Pritchard JK. An expanded view of complex traits: from polygenic to Omnigenic. Cell. 2017;169(7):1177-86.

73. Quinlan AR. BEDTools: the Swiss-Army tool for genome feature analysis. Curr Protoc Bioinformatics. 2014;47:11.12.1-34.

74. Shen L, et al. ngs.plot: Quick mining and visualization of next-generation sequencing data by integrating genomic databases. BMC Genomics. 2014; 15:284.

75. Anders S, Pyl PT, Huber W. HTSeq--a Python framework to work with highthroughput sequencing data. Bioinformatics. 2015;31(2):166-9.

76. Chen J, et al. ToppGene Suite for gene list enrichment analysis and candidate gene prioritization. Nucleic Acids Res. 2009;37(Web Server issue): W305-11.

77. Kurbatova $\mathrm{N}$, et al. PhenStat: a tool kit for standardized analysis of high throughput phenotypic data. PLoS One. 2015;10(7):e0131274.

78. Vorontsov IE, et al. Genome-wide map of human and mouse transcription factor binding sites aggregated from ChIP-Seq data. BMC Res Notes. 2018; 11(1):756.

79. Yevshin I, et al. GTRD: a database on gene transcription regulation-2019 update. Nucleic Acids Res. 2019;47(D1):D100-5.

80. Kulakovskiy IV, et al. HOCOMOCO: expansion and enhancement of the collection of transcription factor binding sites models. Nucleic Acids Res. 2016:44(D1):D116-25.

81. Kulakovskiy IV, et al. HOCOMOCO: a comprehensive collection of human transcription factor binding sites models. Nucleic Acids Res. 2013; 41(Database issue):D195-202.

82. Kuhn M. Building Predictive Models in R Using the caret Package. Journal of Statistical Software. 2008;28(5):26

\section{Publisher's Note}

Springer Nature remains neutral with regard to jurisdictional claims in published maps and institutional affiliations.

\section{Ready to submit your research? Choose BMC and benefit from:}

- fast, convenient online submission

- thorough peer review by experienced researchers in your field

- rapid publication on acceptance

- support for research data, including large and complex data types

- gold Open Access which fosters wider collaboration and increased citations

- maximum visibility for your research: over $100 \mathrm{M}$ website views per year

At BMC, research is always in progress.

Learn more biomedcentral.com/submissions 